\title{
Using a computational domain and a three-stage node location procedure for multi-sweeping algorithms
}

\author{
E. Ruiz-Gironés ${ }^{\mathrm{a}}$, X. Roca ${ }^{\mathrm{a}}$, J. Sarrate ${ }^{*, a}$ \\ ${ }^{a}$ Laboratori de Càlcul Numèric (LaCàN), \\ Departament de Matemàtica Aplicada III, \\ Universitat Politècnica de Catalunya, \\ Jordi Girona 1-3, E-08034 Barcelona, Spain \\ Tel. 34-93-401 69 11, Fax: 34-93-401 1825
}

\begin{abstract}
The multi-sweeping method is one of the most used algorithms to generate hexahedral meshes for extrusion volumes. In this method the geometry is decomposed in sub-volumes by means of projecting nodes along the sweep direction and imprinting faces. Nevertheless, the quality of the final mesh is determined by the location of inner nodes created during the decomposition process and by the robustness of the imprinting process.

In this work we present two original contributions to increase the quality of the decomposition process. On the one hand, to improve the robustness of the imprints we introduce the new concept of computational domain for extrusion geometries. Since the computational domain is a planar representation of the sweep levels, we improve several geometric operations involved in the imprinting process. On the other hand, we propose a three-stage procedure to improve the location of the inner nodes created during the decomposition process. First, inner nodes are projected towards source surfaces. Second, the nodes are projected back towards target surfaces. Third, the final position of inner nodes is computed as a weighted average of the projections from source and target surfaces.
\end{abstract}

Key words: Mesh generation, hexahedral mesh, multi-sweeping, computational domain, geometry decomposition

\footnotetext{
*Corresponding author

Email addresses: eloi.ruiz@upc.edu (E. Ruiz-Gironés), xevi.roca@upc.edu (X. Roca), jose.sarrate@upc.edu (J. Sarrate )
} 


\section{Introduction}

The finite element method has emerged as a fundamental numeric technique in applied sciences and engineering. However, mesh generation still remains as one of the major bottlenecks in the simulation process. Although several automatic algorithms have been developed to generate unstructured tetrahedral meshes $[1,2]$, there is no automatic hexahedral mesher that can handle any kind of geometry. Therefore, specific algorithms have been developed for specific types of geometries that often appear in industrial applications. For instance, sweeping algorithms $[3,4,5,6,7]$ have been successfully applied to extrusion geometries. These algorithms can be applied in extrusion volumes defined by one source and one target surface. For this reason, they are called one-to-one sweeping methods. Unfortunately, real CAD extrusion models are not composed by a single one-toone extrusion volume. Therefore, special attention has been focused on algorithms that automatically decompose any extrusion geometry into these simpler pieces. Many-to-one algorithms [8] can handle extrusion geometries with several source surfaces but a single target surface. These algorithms decompose the initial volume into one-to-one sub-volumes and then discretize each one separately. In the last years, several algorithms appeared to mesh many-to-many extrusion geometries with multiple source and target surfaces $[9,10,11,12]$. These methods split the original geometry into many-to-one sub-volumes. Each sub-volume is further decomposed into one-to-one barrels. The decomposition process is achieved by projecting target surfaces to corresponding source surfaces. In addition, an imprinting process between source and target surfaces is performed to determine the decomposition.

However, the quality of the final mesh obtained by a multi-sweeping method is heavily affected by the robustness of the imprinting process and by the location of inner nodes created during the decomposition. These effects are highly noticeable when the geometry contains high-curved surfaces or non-planar sweep levels.

To overcome these drawbacks, we propose two new procedures that allow us generating high quality decompositions. The first one is designed to increase the robustness of the imprinting process. To this end, we introduce the new concept of computational domain for extrusion geometries. The computational domain is a planar representation of the sweep levels that allows improving the robustness of several geometric operations involved in the imprinting process. Note that all the imprinting operations are performed in the computational domain. Moreover, we also detail an additional procedure that uses this computational domain to project nodes from any target surface that is propagating along the sweep path onto its 
corresponding geometrical source surface. The second procedure is designed to improve the location of inner nodes created during the decomposition process. To this end, we propose a multi-sweeping algorithm in which the decomposition process is performed in three stages: in the first one, the nodes of target surfaces are projected towards source surfaces; in the second stage, the nodes of source surfaces are projected back towards target surfaces; and in the third stage, the final position of inner nodes is computed as the weighted average of the location of nodes projected from source and target faces.

The proposed multi-sweeping method has four additional advantages. First, it allows decoupling the element size of the decomposition process and the element size of the final mesh. The optimal element size for the decomposition process may not coincide with the element size prescribed by the user. Usually, the decomposition element size should be smaller than the discretization size. Thus, better imprints are computed. Second, the parity condition on the surfaces is not imposed during the imprinting time. Instead, this condition is imposed when the geometry is decomposed by solving an integer linear problem. This way, we avoid to impose an even number of intervals on the boundary of the surfaces, and no constraints are added to compute the intersections between edges. This results in better quality imprints. Third, we couple the decomposition process and the meshing process. We realize that the information computed in the decomposition process is required at the discretization time. Hence, during the meshing process, we avoid to recalculate information that was previously computed. Fourth, our implementation does not rely on a virtual geometry engine. Therefore, this method can be successfully implemented using a classical geometry engine. While these advantages are present in other multi-sweeping methods [9, 10], they were not previously contained in a single multi-sweeping algorithm.

\section{The multi-sweeping method}

In this section we illustrate the basic steps of the multi-sweeping decomposition process by applying it to the many-to-many geometry presented in Figure 1. The algorithm starts by classifying the faces of the geometry as target, source or linking sides, see Figure 1(a). Then, the linking sides are meshed using a structured quadrilateral mesh, see Figure 1(b). This mesh provides a set of levels between source and target faces. Next, the process of projecting nodes and imprinting surfaces is started. This process starts at the lower level target surfaces, see Figure 1(c). This face is advanced to the next level using the mesh of the linking sides. This process is iterated until a source face is reached, Figure 1(d). 


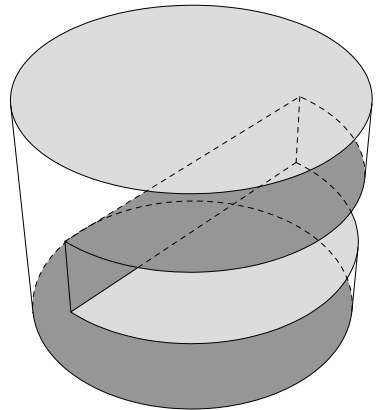

(a)
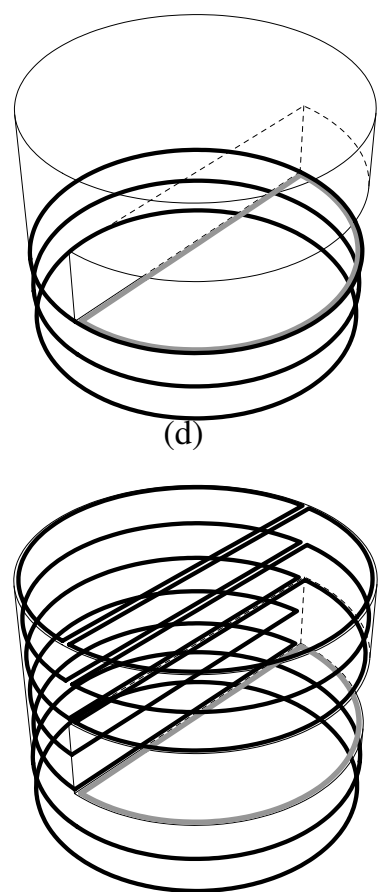

(g)

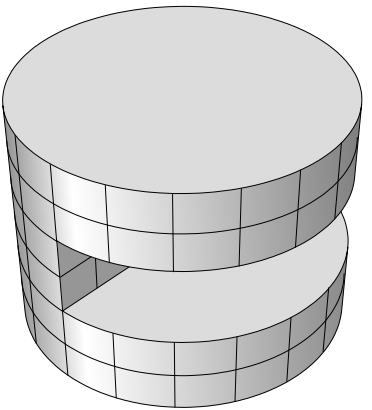

(b)
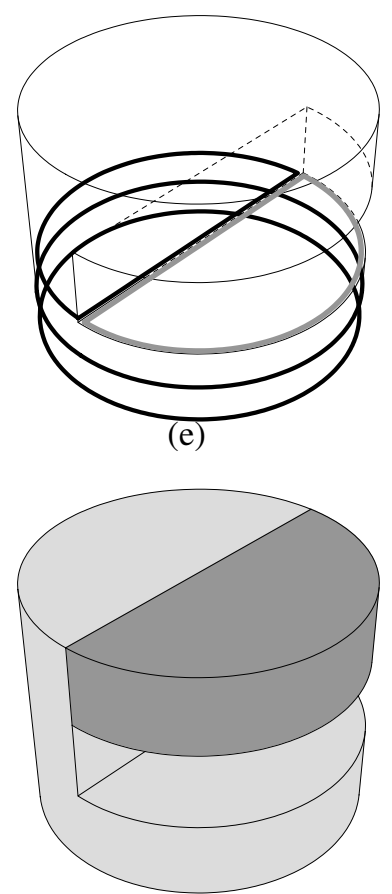

(h)

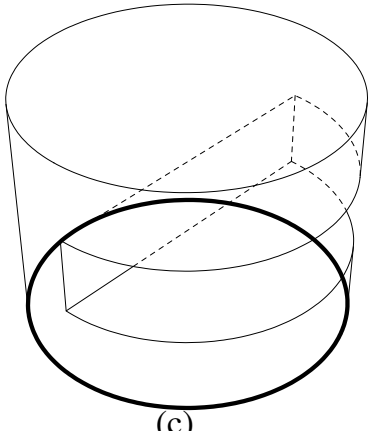

(c)
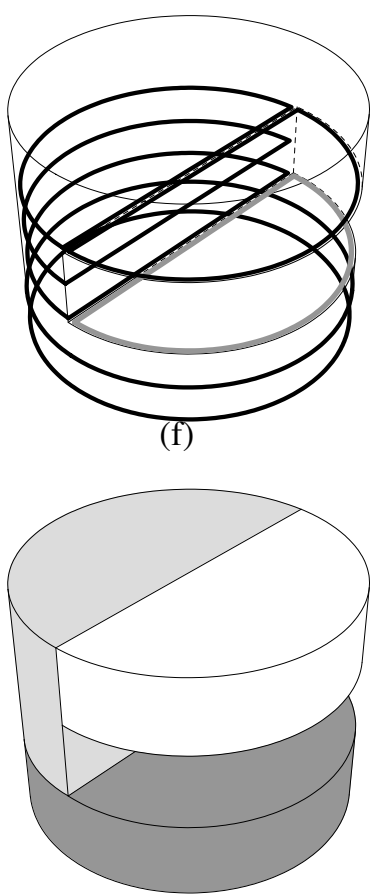

(i)

Figure 1: Summary of the multi-sweeping method. (a) Classification of the surfaces into source (light grey), target (dark grey) and linking sides (transparent). (b) Discretization of the linking sides using submapping. (c) Initial step of the imprinting process at the lowest level target surface. (d) The projection process reaches the first source face. (e) Imprinting process between a source and a target face. (f) Second target face is reached. (g) Result of the projection and imprinting process. (h) Geometry decomposition into a many-to-one and a one-to-one sub-volumes. (i) Geometry decomposition into three one-to-one barrels.

At this point, we have to compute an imprint to determine the section of the target face that will be further propagated. Figure 1(e) shows the result of this imprint. 
The projection process is iterated until a new target face is added at the fifth level, see Figure 1(f). Now, both target faces are projected to the upper levels. When the last source face is reached, the projection and imprinting process ends. As a result of this process, the projected nodes describe the imprints at each level, see Figure $1(\mathrm{~g})$. These imprints determine the decomposition into several sub-volumes. Figure 1(h) shows the geometry decomposed into two sub-volumes. Each one of these sub-volumes is defined by one or more source surfaces and a single target surface. Note that sub-volumes can be also delimited by parts of the source surfaces created during the imprinting process. Finally, each sub-volume is further decomposed into one-to-one barrels, see Figure 1(i).

Taking into account the overview presented in Figure 1, the proposed multisweeping algorithm is composed by four steps. First, the geometry is pre-processed (Section 3). Second, we perform the projection and imprinting process (Section 6). Third, the geometry is decomposed into one-to-one barrels (Section 7). Fourth, we discretize each barrel to obtain the final mesh (Section 8).

To clarify the presentation of the decomposition process, in Section 4 we detail the basic data structures used in our implementation. Moreover, in Section 5 we introduce the new concept of computational domain which allows us improving both the robustness of the imprinting process and the projection of nodes onto geometric source surfaces. Finally, in Section 9 we present several examples that illustrate the capabilities of the proposed method.

\section{Geometry pre-proces}

\subsection{Surface and edge classification}

The first step of the algorithm is the classification of the geometry surfaces. Each surface is classified as source, target or linking side. Source surfaces are the ones that are meshed using quadrilateral elements. The mesh on source surfaces is then projected to the target surfaces. Finally, linking sides are the surfaces that connect source and target surfaces. In order to properly classify the surfaces of the geometry, we have used the technique presented in [13]. The main idea is to find a non-submappable surface (or any arbitrary surface if a non-submappable one does not exist) and classify it as target surface. Then, adjacent surfaces are classified as source, target or linking sides depending on the angle between them and the original one. The algorithm is iterated in an advancing front manner until all the surfaces are classified. Figure 2(a) presents a simple many-to-many extrusion volume with its surfaces properly classified as source, target and linking sides. Once the surfaces of the volume are classified, we proceed to classify the edges 


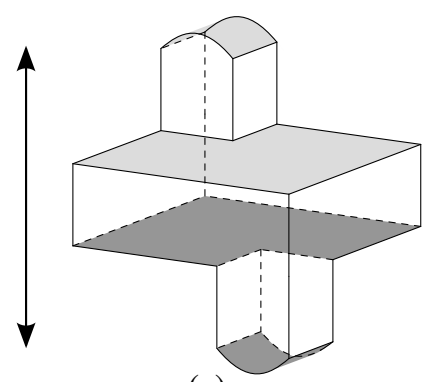

(a)

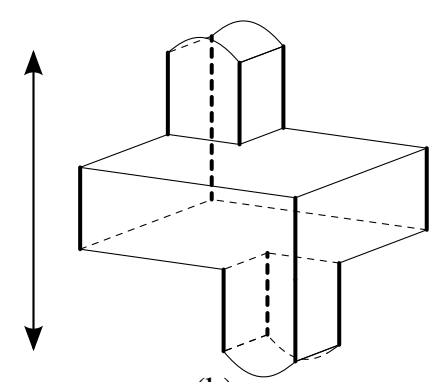

(b)

Figure 2: Classification of surfaces and edges for a simple geometry. (a) Sweep surface classification: source surfaces (light grey), target surfaces (dark grey) and linking sides (transparent). (b) Sweep edge classification: parallel edges (thick line) and transversal edges (thin line).

of the geometry. The edges are classified as parallel or transversal to the sweep direction. Figure 2(b) presents a many-to-many geometry with its edges properly classified as parallel or transversal.

\subsection{Linking sides discretization}

Once the edges and surfaces of the geometry are classified, we mesh the linking sides using the submapping algorithm $[14,15]$. On the one hand, the element size of sweep parallel edges is related to the final mesh element size. On the other hand, the element size of sweep transversal edges is related to the decomposition process element size. This way, we decouple the element size used in the decomposition process and the element size of the final mesh. In general, the element size of the decomposition should be smaller than the element size of the final mesh. This leads to a more accurate imprint and a final mesh with better quality. In our implementation, the default element size of the decomposition is the half of the element size of the final mesh. Figure 3(a) shows the mesh generated on the linking sides of a multi-sweep geometry using the submapping method. In addition, Figure 3(b) shows the mesh generated on the linking sides using the submapping method for the example presented in Figure 1. Note that the structured quadrilateral mesh for the linking sides determines a set of node levels between source and target surfaces.

\section{Data structures}

This section defines three basic data structures that will be used through this work. Although they were previously introduced in [9], we include them for completeness. 


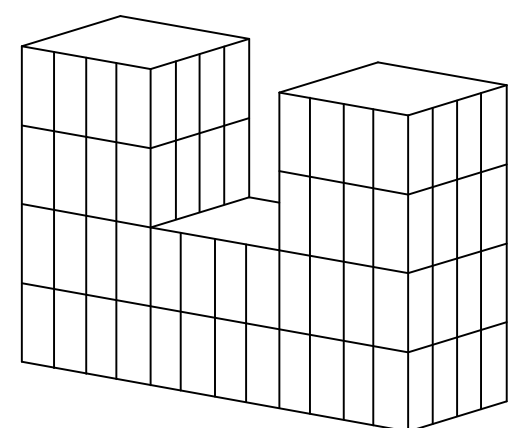

(a)

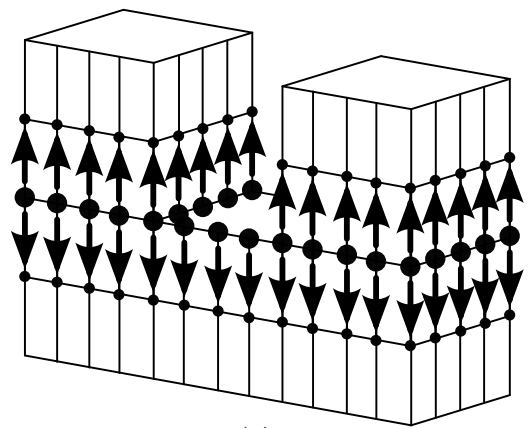

(c)

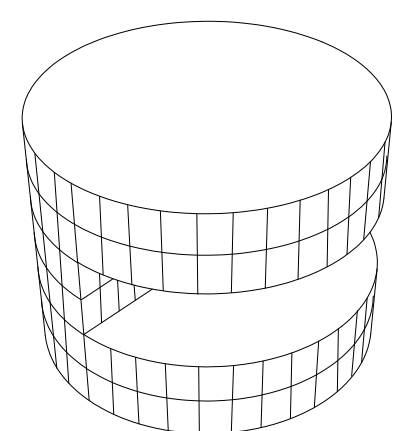

(b)

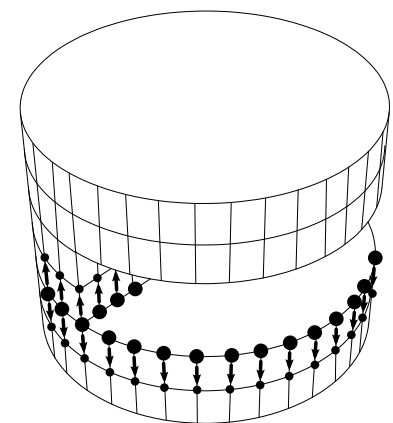

(d)

Figure 3: Two simple extrusion geometries. (a) and (b), meshes generated on the linking sides using the submapping method. (c) and (d), detail of levels of sweep nodes.

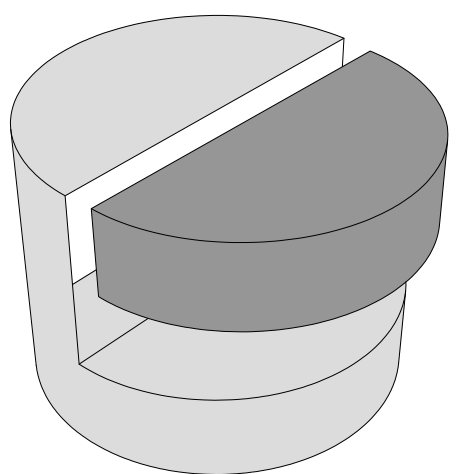

(a)

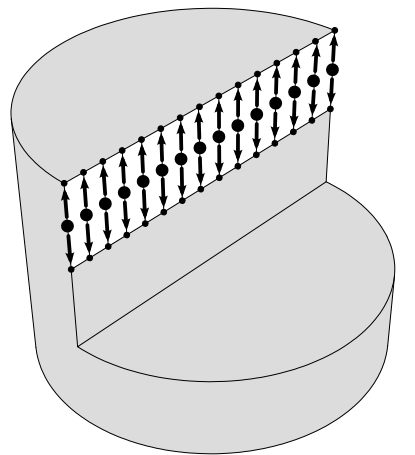

(b)

Figure 4: (a) Decomposition of the geometry into a one-to-one and a many-to-one sub-volumes. (b) Sweep nodes created in the interior of the volume during the decomposition process.

\subsection{Sweep nodes}

Sweep nodes are a computational structure used in the algorithm to store the connectivity of the linking sides mesh along the sweep direction. Each sweep 
node stores two pointers: a pointer to the next sweep node in the sweep direction and a pointer to the previous sweep node in the counter-sweep direction. It is worth to notice that some of these pointers may be null. Figure 3(c) shows an extrusion geometry with a row of sweep nodes. Figure 3(d) shows a row of sweep nodes for the example presented in Figure 1. Note that in these examples there are sweep nodes that do not point to a sweep node in the next or the previous level. As the decomposition algorithm proceeds, new sweeping nodes are created inside the volume. These nodes determine the bounds of the decomposition sub-volumes, see Figure 4(a). For instance, Figure 4(b) shows the sweep nodes created for the multi-sweeping geometry introduced in Figure 1.

\subsection{The loop geometry engine}

In order to decompose the geometry, we represent the source, target and midlevel faces as loops of sweep nodes. To this end, we use the loop geometry engine proposed in [9]. For instance Figure 5(a) shows a circular face represented by: 1) a loop face; 2) a loop wire that describes the boundary of the loop face; 3) an ordered list of two loop edges that defines the loop wire and 4) two loop vertices that define the initial and final points of each loop edge.

Loop vertices are represented by a sweep node. Loop edges are defined by an ordered list of sweep nodes. This list provides a discretization of the loop edge. Loop wires are defined by a closed loop of loop edges. Loop faces are represented by a loop wire that defines the outer boundary and several loop wires that define inner boundaries. In addition, we use the topology graph to describe the topology of the loop faces. Figure 5(b) shows the topology graph of the loop face presented in Figure 5(a). For instance, the loop wire uses loop edge 1 and, conversely, loop edge 1 is included in the loop wire.

Loop geometry engine structure is similar to a geometry engine, except that not all the elements in the loop geometry engine have an underlying geometrical representation. This engine provides both geometrical and topological information about the loop geometry. In addition, this structure is responsible for creating the loop geometry and maintaining the topology graph during the whole decomposition process.

\subsection{Control loops}

Control loops are defined as the loops of sweep nodes that bound each sweep level, large dots in Figure 6. In a many-to-many geometry the number of control loops may differ from level to level. For instance, in Figure 6(b) we show two levels of a many-to-one geometry. Note that the lower level is described by a 

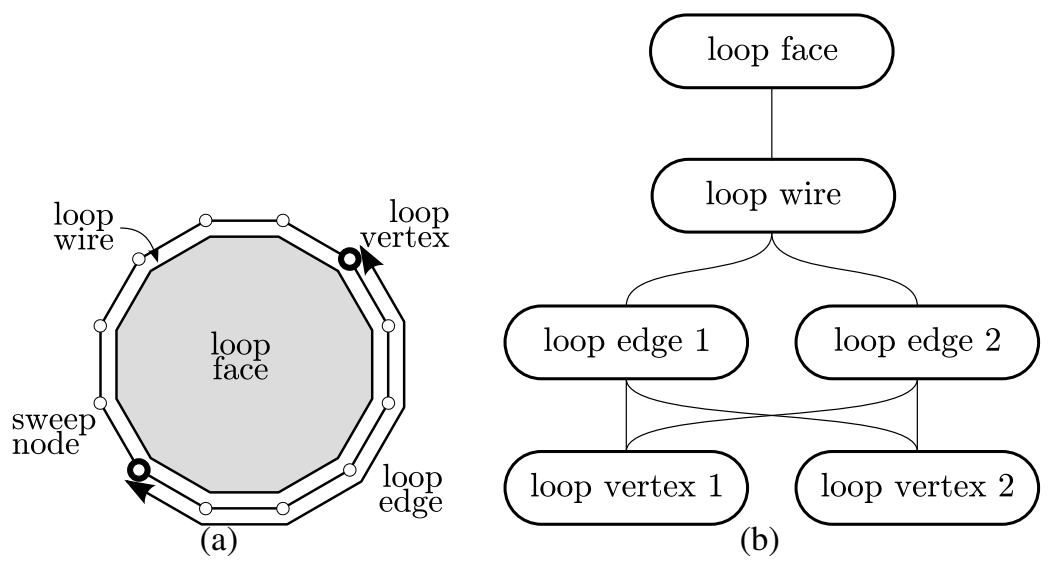

Figure 5: (a) A loop face and (b) its topology graph.
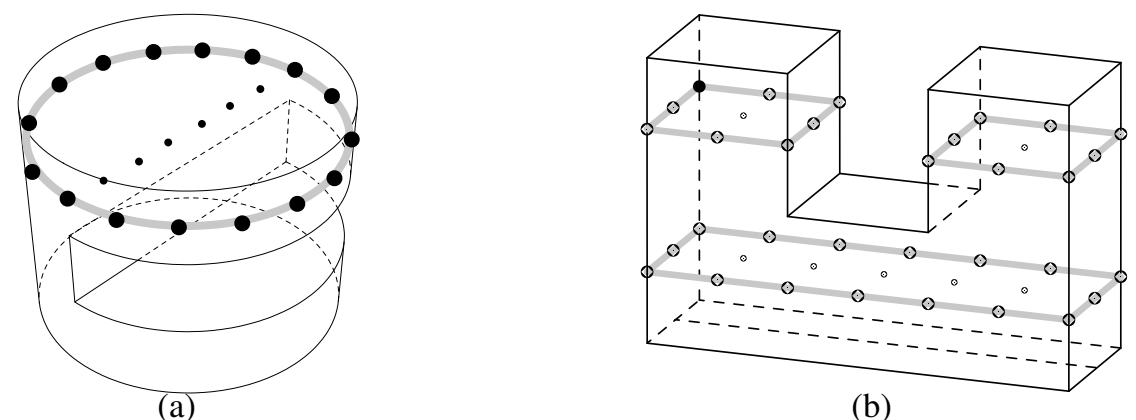

(b)

Figure 6: (a) One control loop in a many-to-many geometry. (b) Three control loops in a manyto-many geometry.

single control loop whereas the upper level is defined by two control loops. Each control loop is composed by a loop of sweep nodes that define the outer boundary of the sweep level and several loops of sweep nodes that define inner boundaries of the sweep level.

Control loops are used to project sweep nodes between two consecutive sweep levels. To this end, they store a list of nodes to project to the next level, small dots in Figure 6.

For each level, there is a set of target loop faces $\left(\tau_{i}\right)_{i=1, \ldots, n}$ and a set of source loop faces $\left(\sigma_{i}\right)_{i=1, \ldots, m}$. Note that mid-level target loop faces may appear as a consequence of the projection process. In this work we propose the following procedure to construct the control loops for the associated target and source loop 

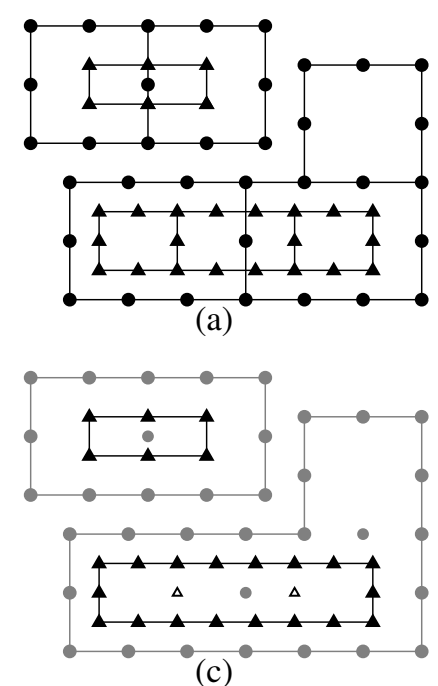
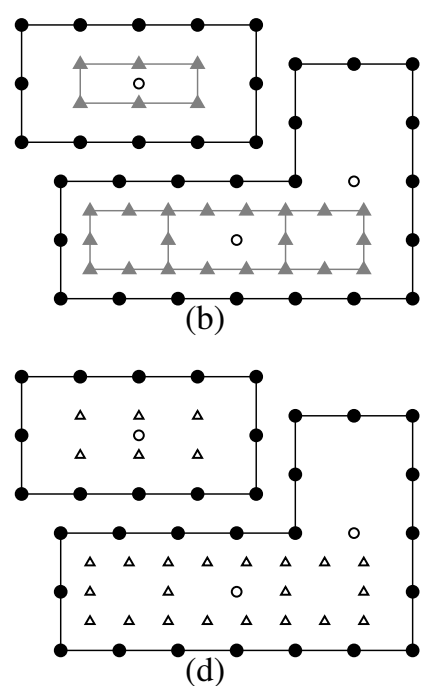

Figure 7: Procedure to construct the control loops. (a) Initial target (black circles) and source (black triangles) loop faces. (b) Union of target loop faces into target control loops. Nodes defining the loops are represented as black circles and nodes to project as white circles. (c) Union of source loop faces into source control loops. Nodes defining the loops are represented as black triangles and nodes to project as white triangles. (d) Insertion of nodes of source control loops into target control loops.

faces:

(i) Compute provisional target control loops, $C L_{t}$, as follows:

- The loops of nodes of control loops are defined as the boolean union $C L_{t}=\bigcup_{i=1, \ldots, n} \tau_{i}$, see [16] for more details about the boolean union.

- The nodes to project are those that belong to more than one loop face and are not contained in the loops of $C L_{t}$.

(ii) Compute provisional source control loops, $C L_{s}$, using the same procedure detailed in step (i).

(iii) Collect the nodes of control loops in $C L_{s}$ that are not included in $C L_{t}$. Then, we insert those nodes in the corresponding control loop in $C L_{t}$.

Note that in the previous procedure we can skip the second and the third steps if there are not source loop faces in the given level. Figure 7 illustrates the process of computing control loops. Figure 7(a) presents a sketch of the previous 
procedure. To this end we consider five target loop faces bounded by black circles in Figure 7(a), and four source loop faces bounded by black triangles in Figure 7(a). The first step of the algorithm is to compute the provisional target control loops, $C L_{t}$, see Figure 7(b). The loops of target control loops are defined using black circles and the nodes to project are defined using white circles. Note that the nodes to project are those that belong to more than one loop face. Next, the provisional source control loops, $C L_{s}$, are computed, see Figure 7(c). In this figure, the loops of nodes are represented using black triangles and the nodes to project are represented using white triangles. Finally, source control loops are inserted in the corresponding target control loops, see Figure 7(d).

\section{The computational domain}

It is important to point out that control loops in real geometries are usually non-planar. Therefore, the imprinting operations needed in the decomposition process may lead to inaccurate representations due to tolerance definitions. To overcome this drawback in this section we introduce the new concept of computational domain.

The computational domain of a given control loop is a planar representation of it. The computational domain is used to: 1.- project the sweep nodes through the volume (see Figure 4(b)); 2.- perform the imprinting between loop faces; and 3.- project sweep nodes onto a geometrical source surface, see Section 6. For instance, in Figure 8(a) we mark with thick black line the curved top surface of the geometry introduced in Section 3.1. Figure 8(b) shows the corresponding curved control loop in the physical domain and its representation in the computational domain.

To obtain a planar representation of control loops we use the pseudo-area and the pseudo-normal vectors considered in [7]. Given a loop of sweep nodes $X=\left\{\mathbf{x}_{i}\right\}_{i=1, \ldots, n}$, we define the pseudo-area vector as

$$
\mathbf{a}:=\frac{1}{2} \sum_{i=1}^{n} \mathbf{x}_{i} \times \mathbf{x}_{i+1},
$$

where $\mathbf{x}_{n+1}=\mathbf{x}_{1}$. If the control loop is defined by $p$ loops of sweep nodes, $X_{1}, \ldots, X_{p}$ the pseudo area vector is defined as

$$
\mathbf{a}:=\mathbf{a}_{1}+\cdots+\mathbf{a}_{p}
$$




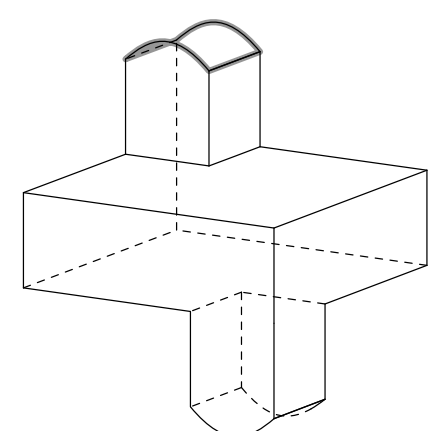

(a)

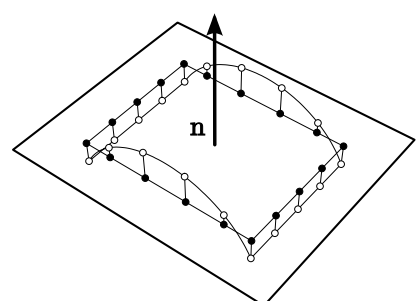

(b)

Figure 8: (a) Geometry with two curved cap surfaces. (b) Physical sweep nodes (white circles) and computational sweep nodes (black circles) of the top curved surface.

where $\mathbf{a}_{1}, \ldots, \mathbf{a}_{p}$ are the pseudo-areas of loops $X_{1}, \ldots X_{p}$, respectively. The pseudo-normal vector is defined as

$$
\mathbf{n}:=\frac{\mathbf{a}}{\|\mathbf{a}\|} .
$$

The construction of the computational domain of a given control loop is performed in the following manner. First, we compute the pseudo-normal vector (3) of the loops nodes that define the control loop. Second, we define the computational position of $\mathbf{x}_{i}$, for $i=1, \ldots, n$ as

$$
\overline{\mathbf{x}}_{i}=\mathbf{x}_{i}-\left\langle\mathbf{x}_{i}, \mathbf{n}\right\rangle \mathbf{n},
$$

where $\langle\cdot, \cdot\rangle$ denotes the dot product. Note that we project all points $\mathbf{x}_{i}$ to the plane defined by the origin of coordinates and the pseudo-normal vector. In [7], it is proved that among all the possible planar projections of the loop of points, this one maximizes the area of the projected loop. From the 3D representation of the computational domain given by (4), it is straightforward to compute a 2D representation, in $(\xi, \eta)$ coordinates, of the computational domain, see details in Section 6.3.

Two remarks on the proposed method have to be made. First, each control loop has its own computational domain, even when control loops are located in the same sweep level. Second, in order to construct the computational domain, we need to compute the pseudo-normal vector. This vector can be defined if a control loop is set. Therefore, our method can be applied to all extrusion geometries such that all levels are bounded by control loops. Note that periodic surfaces such 
as cylinders or spheres that expand from inside to outside, or vice versa, do not meet this condition. Thus, the proposed method can not be applied to these cases because the control loop is not set. In these situations, we can always split the geometry in two or more sub-volumes such that the control loops are properly defined.

\section{Projection and imprinting process}

The objective of this section is to describe the process of projecting and imprinting loop faces. This leads to the decomposition of the volume into one-to-one barrels. The process of projection and imprinting is performed in three stages. First, we project through the volume the target loop faces towards the source loop faces. In each sweep level, an imprinting process is performed if necessary. Once the source loop faces are reached, it is possible to decompose the volume. However, the location of the sweep nodes created in the first stage can be improved. For this reason, in the second stage, we project back the sweep nodes on source loop faces to target loop faces. In the third stage, the final location of inner sweep nodes is calculated as the weighted average between the locations of sweep nodes projecting from target surfaces and projecting from source surfaces.

\subsection{Loop face projection}

This section is focused on the loop face projection performed during the decomposition process. We start the projection process at the first sweep level. We collect all the target surfaces and compute the associated loop faces as detailed in Section 4.2. Then, the corresponding control loops are calculated according to Section 4.3. Note that there are no source surfaces on the first level. Once the control loops are constructed, the algorithm proceeds to calculate their associated computational domain, see Section 5. The next step is to project the inner sweep nodes to create the next level loop faces. In order to project the sweep nodes between levels we use the sweeping scheme presented in [6, 7]. It is based on a least-squares approximation of an affine mapping defined between two consecutive control loops. Note that the projection of sweep nodes is performed both in the physical domain and the 3D representation of computational domain. The physical and the 3D computational locations are stored for each sweep node. When sweep nodes are projected, the new sweep nodes are connected to the ones of the previous level. The loop faces of the next level are created using the nodes of the next level pointed from the nodes of the current level. In addition, we also create the loop vertices, loop edges and loop wires in the same manner. Finally, the loop 


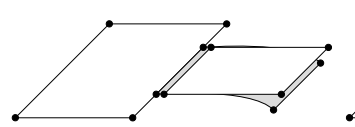

(a)

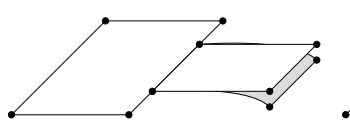

(b)

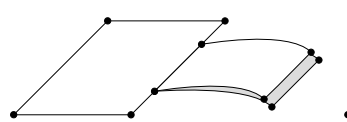

(c)

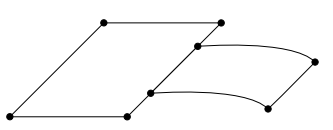

(d)

Figure 9: (a) Two target loop faces (white) and a source loop face (grey). (b) Matching of target loop edges. (c) Projection of target sweep nodes on source faces. (d) Collapse of target sweep nodes and source sweep nodes.

geometry engine is updated in order to reflect the new changes of the loop geometry. During the loop face projection process, the new loop faces created in the next sweep level are considered as target loop faces. At the next level, we collect all the target and source loop faces and update the corresponding control loops. Finally, if it is required an imprinting process is performed for each resulting control loop.

\subsection{Loop face imprinting pre-process}

We have to pre-process the source and target loop faces before starting the actual imprinting process. The loop face imprinting pre-process is performed in the computational domain and then, the physical domain is updated accordingly. To visualize the imprinting pre-process Figure 9 presents the updated physical domain after each one of the imprinting pre-process steps. Figure 9(a) shows two target loop faces (white) and one source loop face (grey). First, the loop edges of target loop faces are matched each other in the 3D representation of the computational domain to ensure a conformal loop geometry, see Figure 9(b). Second, we project the sweep nodes of target loop faces on the geometrical surface of source loop faces, see Figure 9(c). This step is performed using the 2D representation of the computational domain as detailed in Section 6.3. Third, we search for a sweep node in a target loop face and a sweep node in a source loop face that are closer than a given tolerance in the 3D representation of the computational domain. Then, we move the target sweep node to the location of the source sweep node in order to collapse them, see Figure 9(d). This tolerance is defined as $h / \lambda$, where $h$ is the decomposition element size and $\lambda$ has the default value of 5 .

\subsection{Mapping of sweep nodes from the computational domain to source surfaces}

This section is focused on a new procedure to project sweep nodes of a target loop face onto the geometrical surface that defines a source loop face. Note that both target and source loop faces belong to same control loop. For a given source loop face, $\sigma$, we need to detect which nodes of target loop faces lie inside $\sigma$. Since 


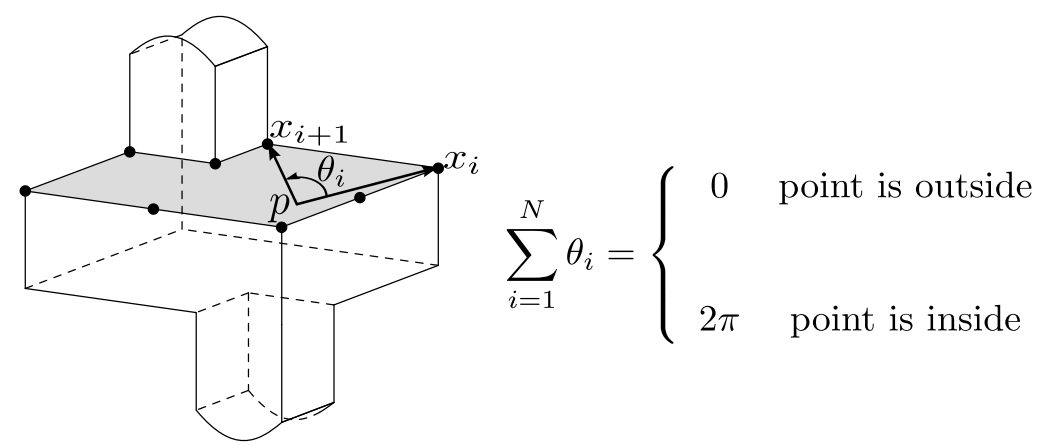

Figure 10: Graphical representation of the winding number algorithm.

loop faces are planar in the computational domain we use the winding number algorithm [17], see Figure 10 for a graphical representation of this algorithm. Given a test point, $p$, the algorithm adds the angles between $p$ and all two consecutive points in the loop. If the result is equal to 0, the point is outside. Else, if the result equals $2 \pi$, the point is inside. That is, the algorithm counts the number of turns around the test point. This algorithm is robust when dealing with planar loops of nodes. Note that in 3D, the number of turns is not well defined. Therefore, the winding number algorithm may fail.

Given a list of sweep nodes inside a source loop face, we have to project them on the surface that defines the source loop face. Note that such nodes come from target surfaces of previous levels. Several algorithms have been developed to project nodes onto a given surface. Most of them involve an orthogonal projection of nodes onto the target surface. Note that these projections are expensive from a computational point of view since it is necessary to solve a root finding problem for each node to project. To overcome this drawback, we propose to use a method previously developed to map meshes between two surfaces [5]. Let $(\xi, \eta)$ and $(x, y, z)$ be the coordinate systems of the $2 \mathrm{D}$ representation of the computational domain and of the physical domain, respectively. Note that $(\xi, \eta)$ coordinates are not univocally defined. For instance, we can apply a planar rotation to obtain another valid coordinate system. However, the procedure to project the nodes to source surfaces does not depend on which coordinate system is selected. Algorithm 1 presents a procedure to compute the $2 \mathrm{D}$ coordinates of a computational sweep node from its 3D coordinates in the computational domain. First, the algorithm finds an orthogonal unitary vector, $\mathbf{e}_{1}$ to the pseudo-normal vector $\mathbf{n}$ that defines the plane of the computational domain (Lines 2-13). The second vector, $\mathbf{e}_{2}$, is calculated as the cross product of $\mathbf{e}_{1}$ and $\mathbf{n}$ (Line 14). Using vectors $\mathbf{e}_{1}$ and 


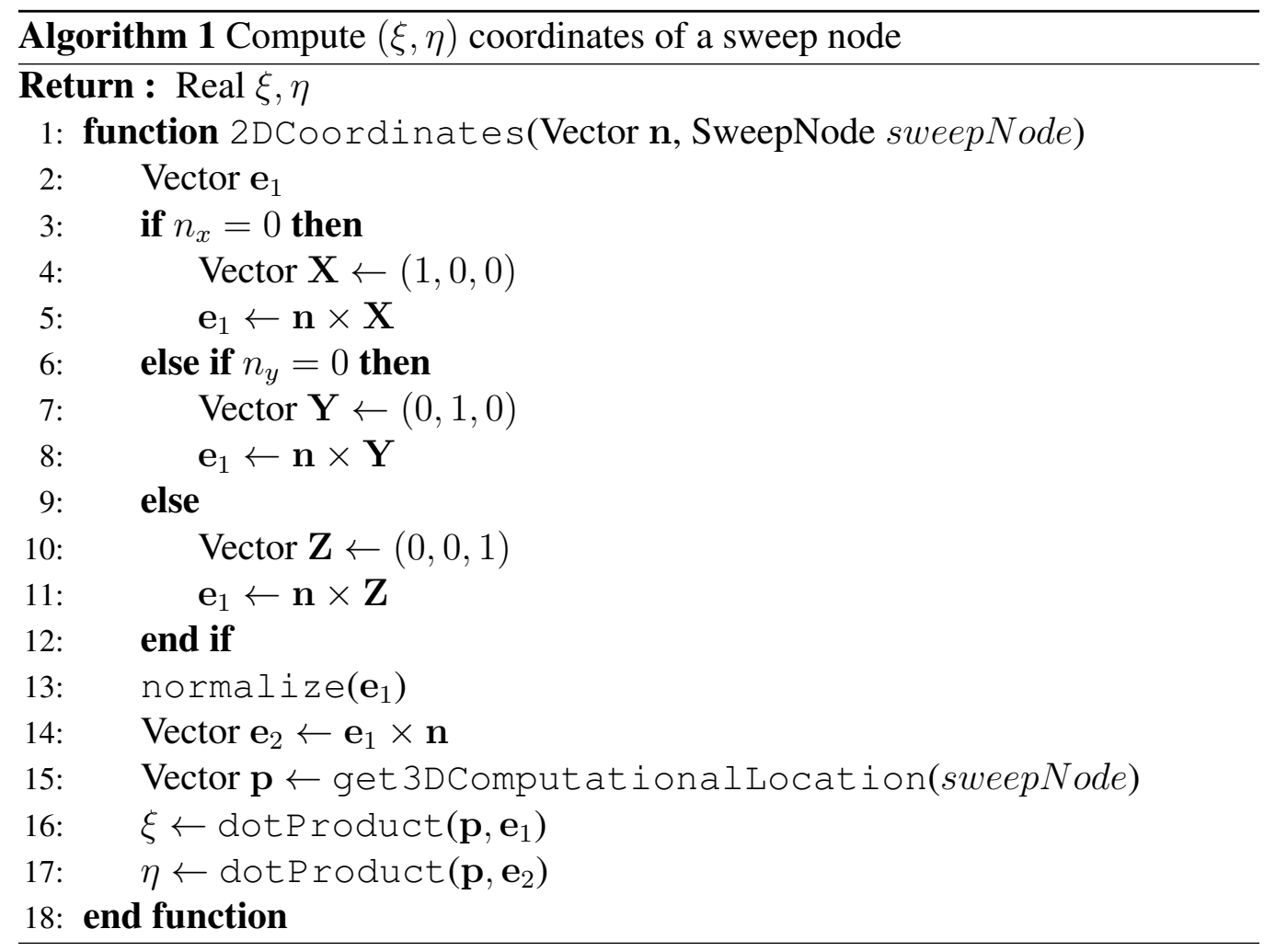

$\mathbf{e}_{2}$, the $(\xi, \eta)$ coordinates are computed (Lines 16-17).

When the 2D coordinates of the sweep nodes are obtained, we have to calculate a mapping, $\boldsymbol{X}(\xi, \eta)$, to project the computational nodes to the physical domain, see Figure 11. Note that source loop faces represent a geometric surface. Thus, instead of projecting the nodes directly to the physical surface, we propose to compute a mapping, $\Pi(\xi, \eta)$, to map the nodes to the parametric domain of the surface. Then, using the parametrization of the surface, $\varphi(u, v)$, we map the corresponding nodes to the physical domain. That is,

$$
\boldsymbol{X}=\varphi \circ \Pi \text {. }
$$

Note that the mapping $\Pi(\xi, \eta)$ is unknown. In this work we propose to approximate $\Pi(\xi, \eta)$ by means of a least-squares approximation of an affine mapping, $\widetilde{\Pi}(\xi, \eta)$, defined between the 2D representation of the computational domain and the parametric domain of the surface, see [5]. That is, we approximate $\boldsymbol{X}(\xi, \eta)$ by

$$
\boldsymbol{X} \approx \varphi \circ \widetilde{\Pi} \text {. }
$$




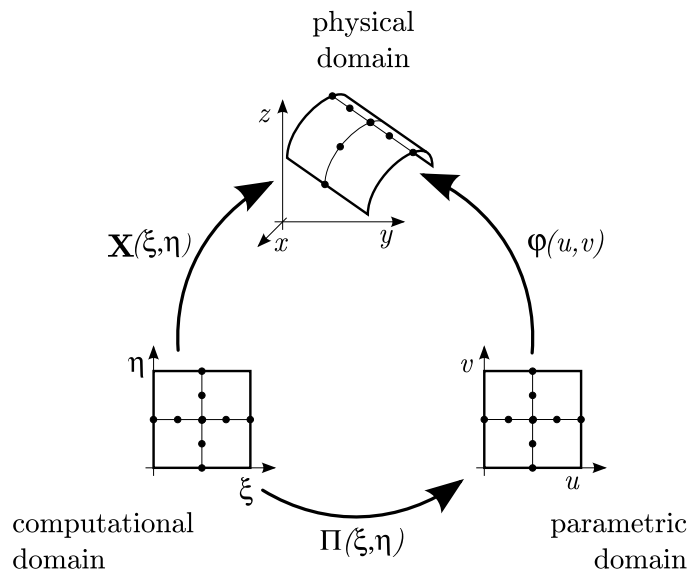

Figure 11: Mapping of computational sweep nodes to source faces.

\subsection{Loop face imprinting}

Once the loop faces are pre-processed, we are ready to perform the imprinting. The result of the imprinting process between target loop faces, $\left(\tau_{i}\right)_{i=1 \ldots n}$, and source loop faces, $\left(\sigma_{i}\right)_{i=1 \ldots m}$, is stored in three lists of loop faces, $\Theta_{o}, \Theta_{t}$ and $\Theta_{s}$. List $\Theta_{o}$ contains the loop faces that came from intersections of a target and a source loop face. The loop faces in $\Theta_{o}$ are called overlap loop faces. List $\Theta_{t}$ contains the sections of target loop faces that do not intersect a source loop face. This list contains the new target loop faces that replace the old target loop faces, $\left(\tau_{i}\right)_{i=1 \ldots n}$. Finally, list $\Theta_{s}$ contains the section of source loop faces that did not intersect a target loop face. This list contains the new source loop faces that replace the old source loop faces, $\left(\sigma_{i}\right)_{i=1 \ldots m}$.

The loop face imprinting operation is based on segment intersections, see $[9,16]$ for more details. The main difference of the presented method is that the imprints are performed in the $3 \mathrm{D}$ representation of the computational domain. Hence, the imprinting process becomes more accurate and robust since skew lines (neither parallel nor concurrent) do not exist in a bi-dimensional space. Therefore, computing the intersection of segments in the computational domain is less affected by tolerance errors than in the physical domain.

In addition, we create a new graph that relates each of the new loop faces with the old loop faces from which they came from. This graph is denoted as loop face partitioning graph. It allows recovering the set of loop faces in which a given loop face is decomposed during the imprinting process. In addition, it also allows recovering the loop faces in which a given loop face is included. Figure 


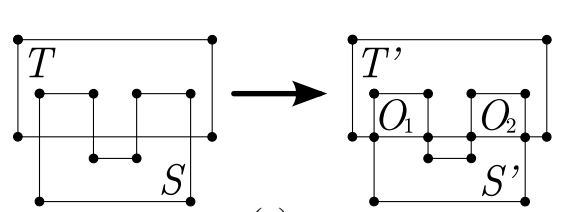

(a)

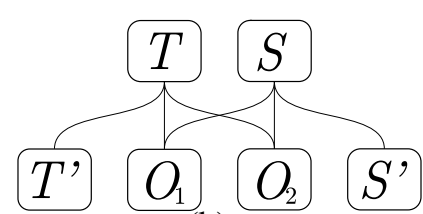

(b)

Figure 12: Imprinting process of a target loop face $(T)$ and a source loop face $(S)$. (a) Imprint process. (b) Loop face partitioning graph.

12(a) shows the imprinting process of a target loop face, $T$, and a source loop face, $S$. In this example, the results of the imprinting are $\Theta_{o}=\left\{O_{1}, O_{2}\right\}, \Theta_{t}=$ $\left\{T^{\prime}\right\}=\{T-S\}$ and $\Theta_{s}=\left\{S^{\prime}\right\}=\{S-T\}$. Figure 12(b) presents the associated partitioning graph. In this case, the old target loop face, $T$, is composed by the new loop faces $T^{\prime}, O_{1}$ and $O_{2}$. Moreover, the new overlap loop face $O_{1}$ is a common section of the old target loop face $T$ and the old source loop face $S$. This information is required to perform the final decomposition process and the discretizacion of all one-to-one barrels.

In order to illustrate the differences between the imprinting process in the physical and computational domain, Figure 13(a) shows a curved loop face, $T$, and a planar loop face, $S$ in the physical domain. The intersection between the segments that define these loop faces in the physical domain are marked using a white circle. Figure 13(b) shows the Boolean difference between $S$ and $T$ computed in the physical domain. Note that we do not obtain the desired result. Figure 13(c) shows the representation of the previous loop faces in the computational domain. When the intersections are calculated in the computational domain, two additional nodes are obtained. Therefore, we obtain the correct representation of $S-T$, see Figure 13(d).

Once the imprinting process is finished, we collect the new target loop faces, $\Theta_{t}$, in order to create the new control loops. These control loops allow projecting the new target faces to the next level. When a loop face is projected, it stores a pointer to the new loop face. Moreover, the new loop faces also stores a pointer to the original one. This way, we establish a relationship between the loop faces over the different sweep levels. This structure is denoted as loop face stack graph. This graph is used both in the decomposition and discretization processes.

The projection and imprinting process is iterated over the sweep levels and it ends when the last level is reached. Figure 14(a) presents a wire frame model of a many-to-many extrusion geometry with two source surfaces and two target surfaces. Figure 14(b) shows its corresponding loop faces when the imprinting 


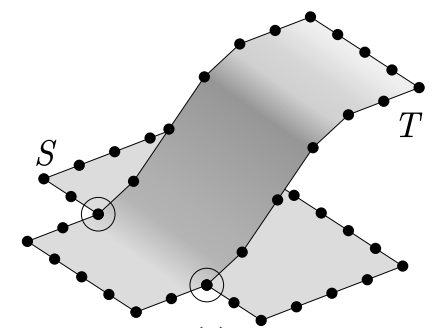

(a)

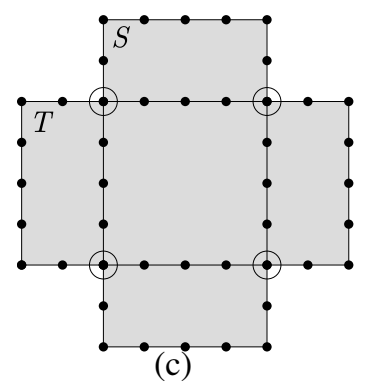

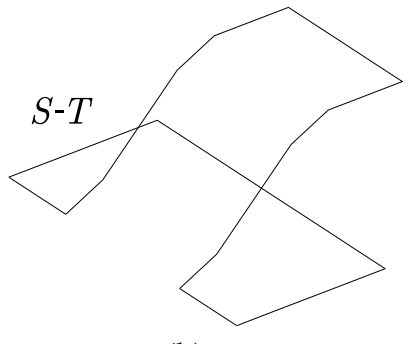

(b)

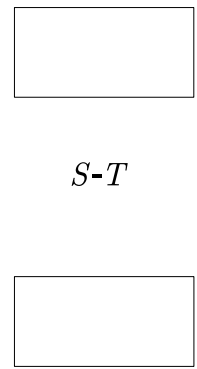

(d)

Figure 13: Boolean difference of loop faces $S$ and $T$. (a) Segment intersections computed in the physical domain. (b) Boolean difference $S-T$ performed in the physical domain. (c) Segment intersections computed in the computational domain. (d) Boolean difference $S-T$ performed in the computational domain.

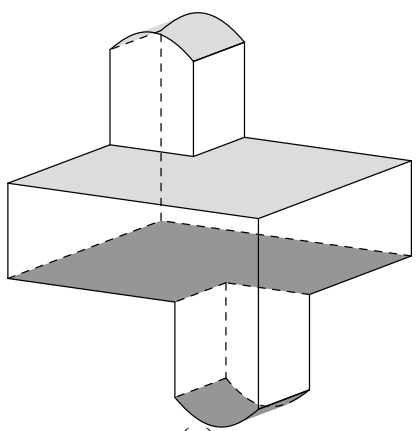

(a)

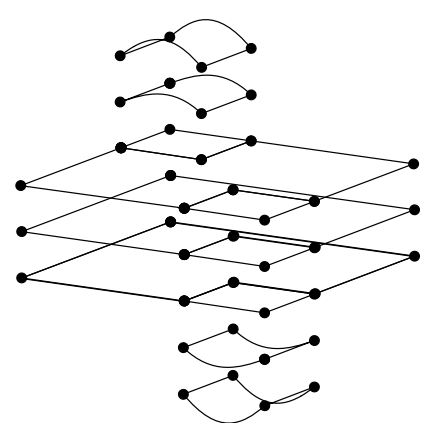

(b)

Figure 14: A many-to-many extrusion geometry and its corresponding loop faces. (a) Wire frame model showing two target surfaces (dark grey) and two source surfaces (light grey). (b) Loop faces.

process ends. Note that the upper surfaces are split due to the diagonal edge in the lower surfaces. 


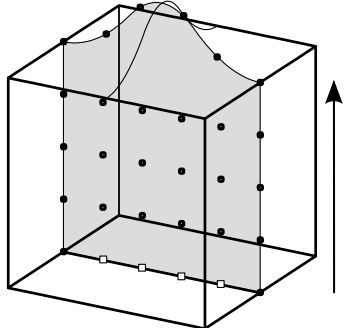

(a)

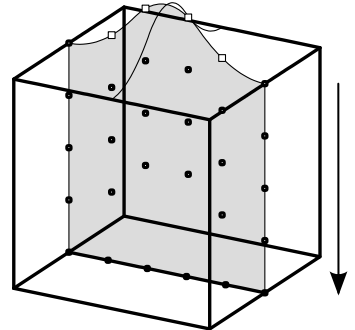

(b)

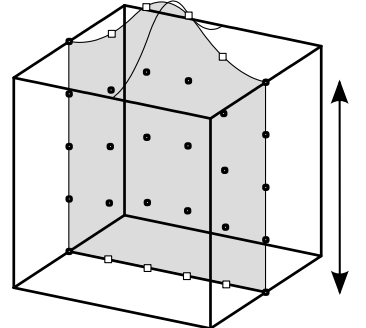

(c)

Figure 15: Projection of inner nodes in a many-to-many geometry. Geometry edges are marked with thick lines. (a) Projecting from target surface. (b) Projecting from source surface. (c) Weighted average between projections from both cap surfaces.

\subsection{Final location of inner sweep nodes}

Once the process of projecting and imprinting ends, we have to improve the position of inner nodes created during this process. To this end, we project the nodes on source surfaces back to target surfaces. Then, the final position of inner nodes is the weighted average of the location of nodes created projecting them from target and source surfaces. Note that this idea has been previously introduced during the meshing process of one-to-one barrels $[10,6]$

For each overlap loop face, $\omega$, obtained during the imprinting process, the nodes that have to be projected to target surfaces are those in $\omega$ such that their previous node lies inside the volume, but not at the boundary. Figure 15 presents a many-to-many extrusion geometry with a planar bottom surface split in two patches (classified as target), and a curved top surface (classified as source). Sweep nodes on cap surfaces that have to be projected through the volume are marked using white squares. Figure 15(a) shows the position of inner nodes projected from target surfaces. These nodes do not capture the shape of the source surface. On the contrary, Figure 15(b) presents the location of inner nodes projected from source surfaces. The nodes do not reproduce the shape of the target surfaces. However, Figure 15(c) presents the node locations by averaging the position of nodes projected from target and source surfaces. Note that the nodes reproduce the shape of both cap surfaces.

For each overlap loop face, $\omega$, and the list of nodes to be projected, nodes, Algorithm 2 presents a procedure to relocate inner nodes. First, at Line 4, the algorithm finds a source control loop, $C L_{s}$, and a target control loop, $C L_{t}$, that define the projection between the two levels (see Algorithm 3). Note that Algorithm 3 updates the initial overlap loop face, $\omega$. Then, at Line 5 of Algorithm 


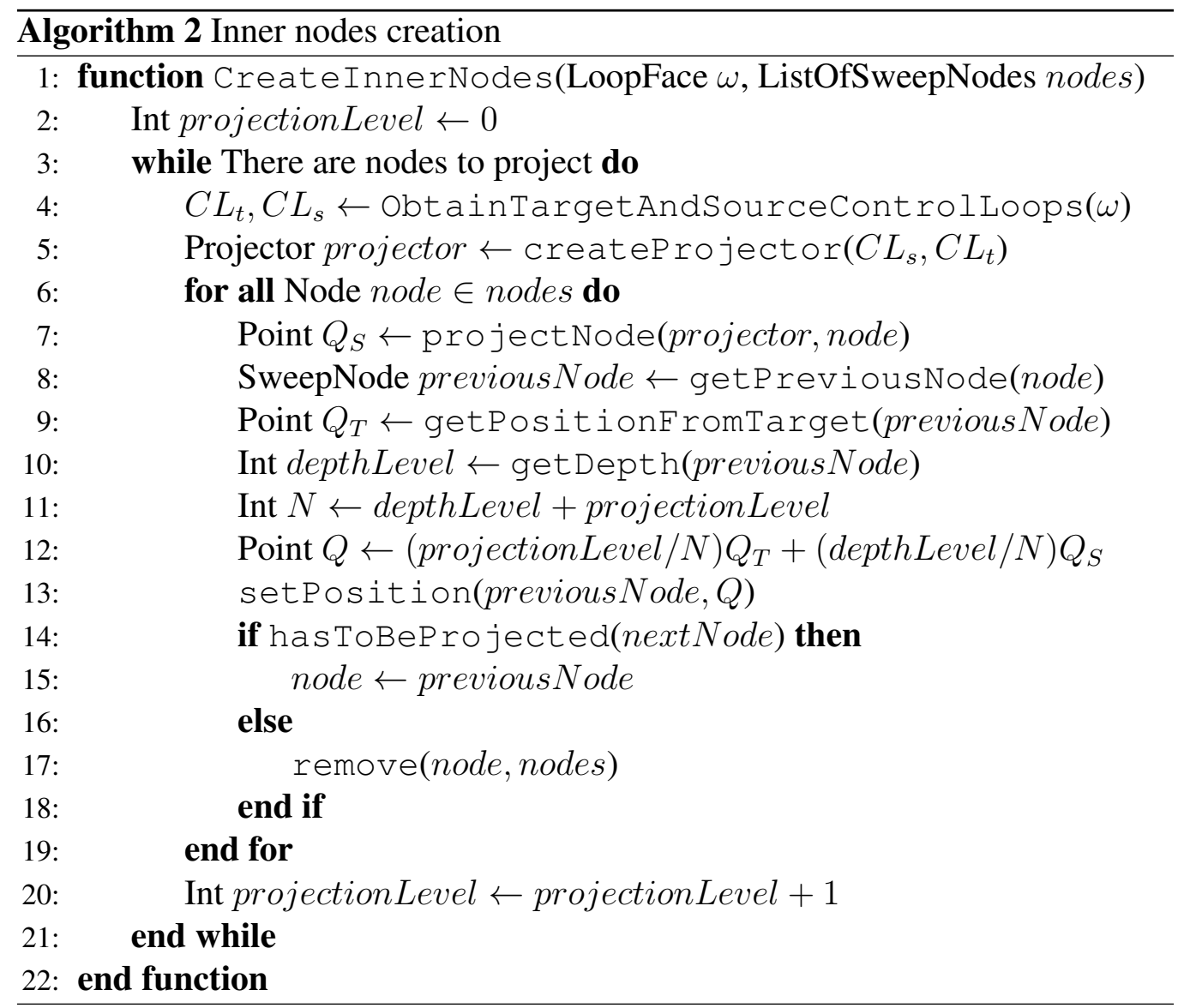

2 , the node projector is constructed as detailed in $[6,7]$. Next, for each node in nodes, the algorithm computes the projection from the source surface, Line 7. Then, at Lines 8 and 9, the previous node and its position computed projecting from target surfaces is recovered. Next, at Line 12 the final position of the previous node is obtained by a weighted average of the computed location projecting from the target and source surfaces. The depth level of a sweep node is defined as the number of times this node has been projected from a target loop face. Finally, the algorithm checks if the previous node has to be projected, Line 14. If so, the node is updated. Else, the node is removed from the list of nodes to project. The procedure is iterated until all nodes are re-located in the physical domain. 

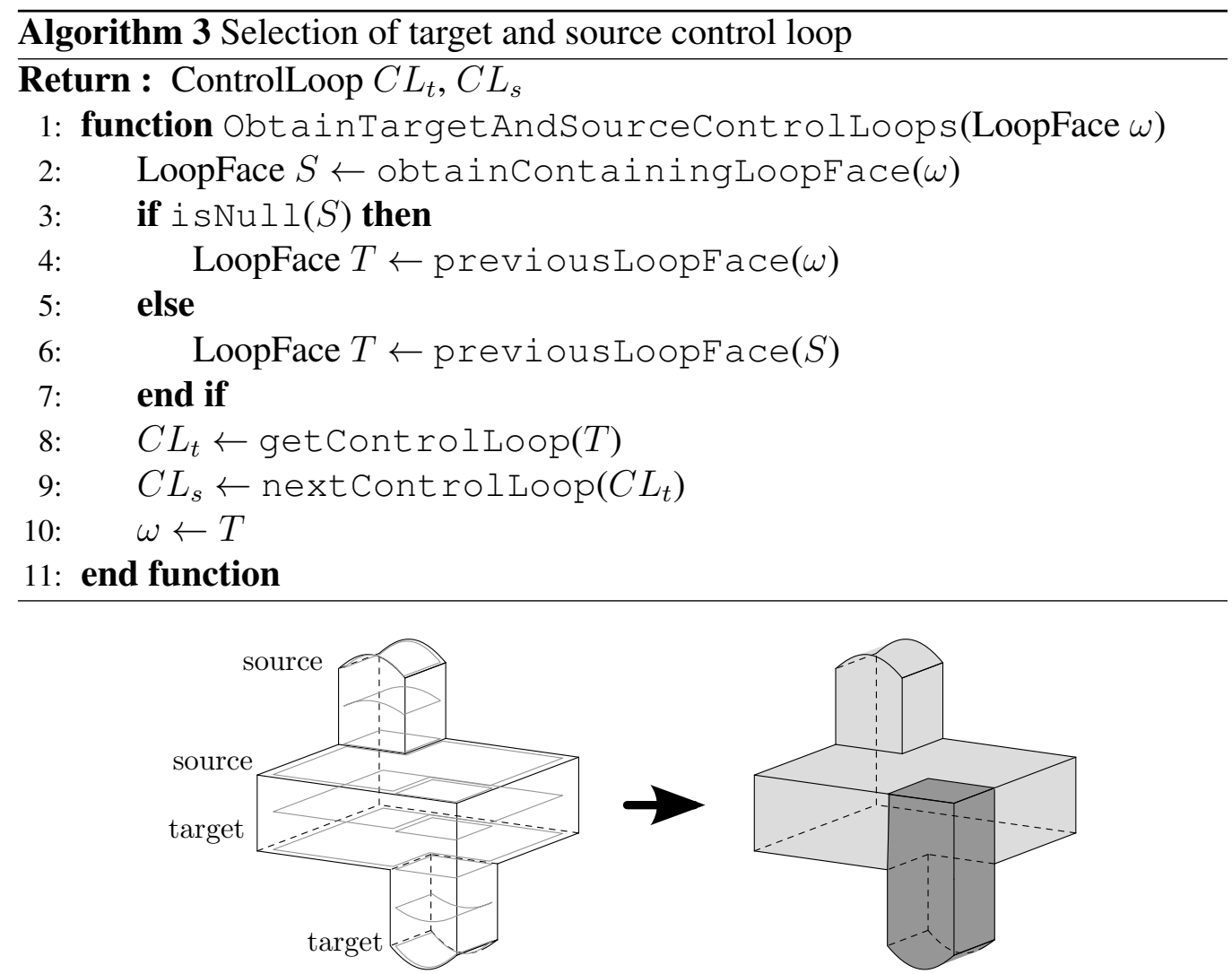

Figure 16: Many-to-many extrusion volume decomposed into a many-to-one and a one-to-one sub-volumes.

\section{Volume decomposition into one-to-one sweep barrels}

The final decomposition process of the geometry into one-to-one barrels is accomplished in two steps. First, the geometry is decomposed into many-toone sub-volumes. Second, each sub-volume is further decomposed in one-to-one sweep barrels. The first step of the decomposition process is straightforward: for each target loop face, we collect every loop face stacked on it. Figure 16 presents a geometry decomposed into two sub-volumes. Note that each sub-volume only contains a single target loop face.

When the geometry is split, we proceed to further decompose each sub-volume separately into barrels in which a one-to-one sweep scheme is applied. It is important to point out that, in order to generate the final mesh in each sub-volume, it is necessary to discretize each barrel in a given order: barrels that are closer 
to source surfaces are meshed first and barrels closer to the target surfaces are meshed later. That is, the meshing process starts at source surfaces and ends at target surfaces. Algorithm 4 presents a method that decomposes a sub-volume into barrels and properly sorts the resulting barrels. Given a sub-volume, the algorithm starts at its target loop face, $\tau$. A loop face stack $\mathcal{T}$ is initialized with $\tau$. Then, each loop face in $\mathcal{T}$ is processed and deleted from the loop face stack $\mathcal{T}$. Given the first loop face $\tau$ in $\mathcal{T}$, we find the first partitioned loop face $\sigma$ stacked over $\tau$. This operation is accomplished using the loop face stack graph. Next, a barrel is created using loop faces $\sigma$ and $\tau$ and it is inserted at the beginning of the list of barrels. Finally, we collect all the containing loop faces in $\sigma$ and proceed to insert them in the loop face stack $\mathcal{T}$. In order to perform this step, we used the loop face partitioning graph. The whole process is iterated until there are no loop faces in the loop face stack $\mathcal{T}$. Figure 17 presents a many-to-one sub-volume decomposed into two barrels.

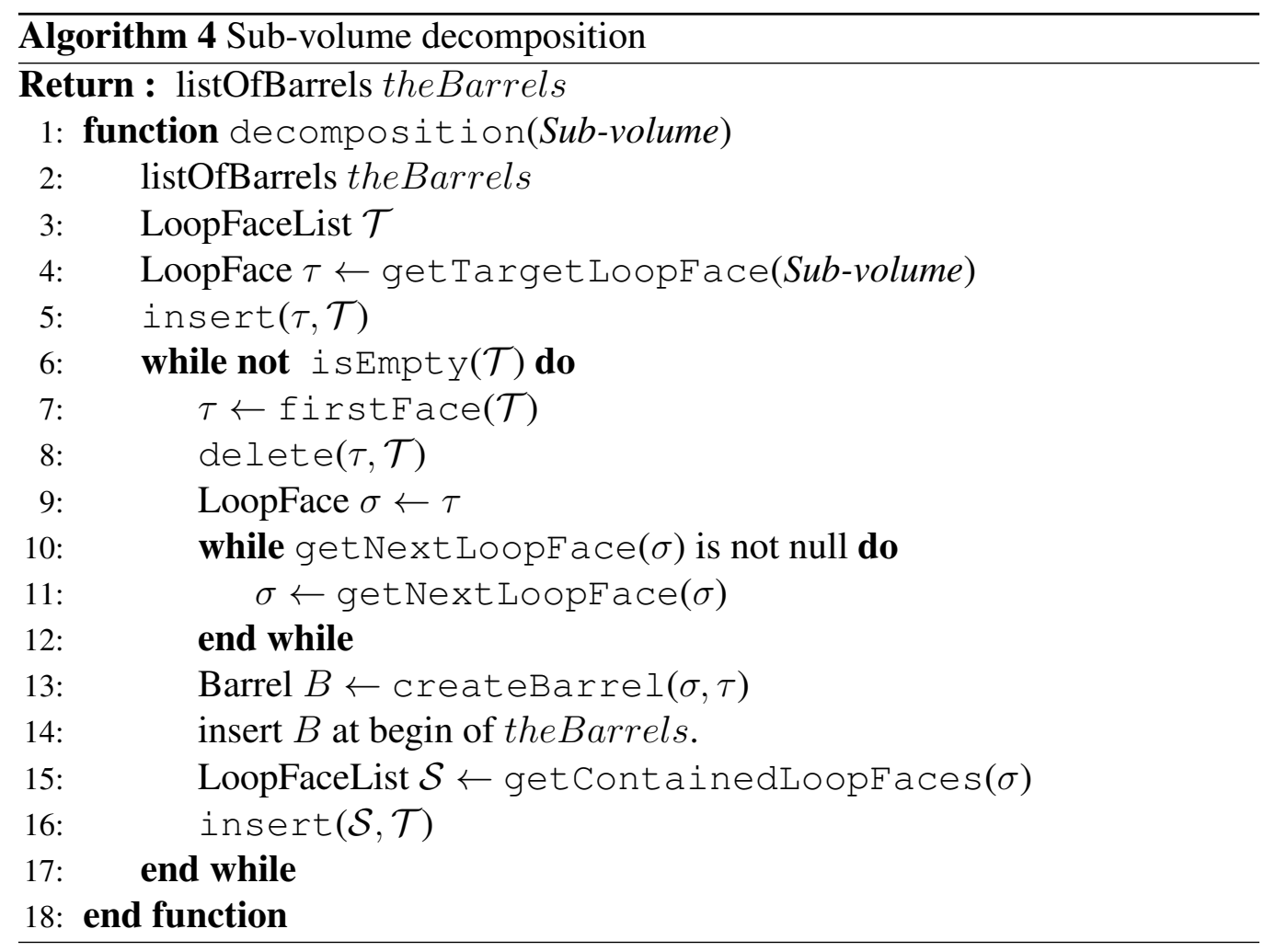




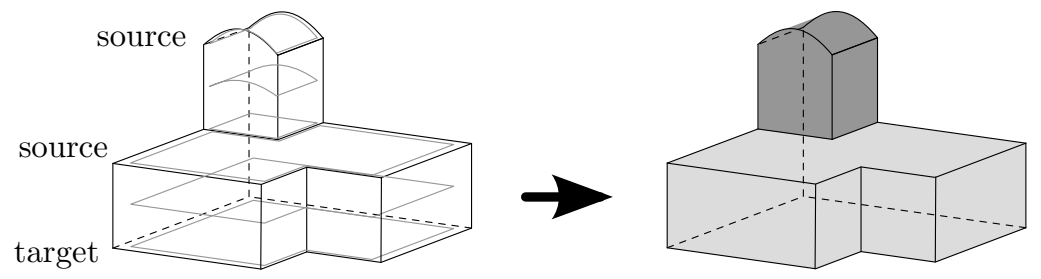

Figure 17: Many-to-one sub-volume decomposed into two barrels.

\section{Geometry discretization}

\subsection{Loop edges discretization}

Prior to the one-to-one barrel discretization, it is necessary to re-mesh the loop edges in order to ensure that each loop face contains an even number of intervals. To this end, we solve an integer linear problem. See [18] for more details about integer linear programming. However, applying this strategy to all loop edges leads to a large integer linear problem, especially for small element sizes in the sweep direction. To reduce the computational cost of the integer linear problem, we impose an even number of intervals only to the source loop faces obtained during the imprinting process. Then, the information is propagated in the sweep direction. Hence, the integer linear problem is

$$
\begin{array}{ll}
\min \sum_{\substack{e \in \mathcal{E} \\
\text { constrained to: }}} n_{e} & \\
\sum_{e \in \sigma} n_{e}=2 n_{\sigma} & \text { for all source loop face } \sigma, \\
n_{e} \geq N_{e} & \text { for all source loop edges } e \in \mathcal{E},
\end{array}
$$

where $n_{e}$ is the number of intervals of loop edge $e, \mathcal{E}$ is the set of loop edges contained in source loop faces, $N_{e}$ is a lower bound for $n_{e}$ and $2 n_{\sigma}$ is the number of intervals of loop face $\sigma$. Note that the unknowns of the ILP (5) are $n_{e}$ and $n_{\sigma}$. In our implementation the default value for $N_{e}$ is $l_{e} / h$ where $l_{e}$ is the length of loop edge $e$ and $h$ is the prescribed element size of the final mesh. When the solution is found, the number of intervals of each source loop face $\sigma$ is even. In order to solve this integer linear program, we use the lpsolve library [19]. Finally, we re-mesh each source loop edge according to the number of intervals specified by the integer linear problem and then we propagate the new nodes in the sweep direction. 


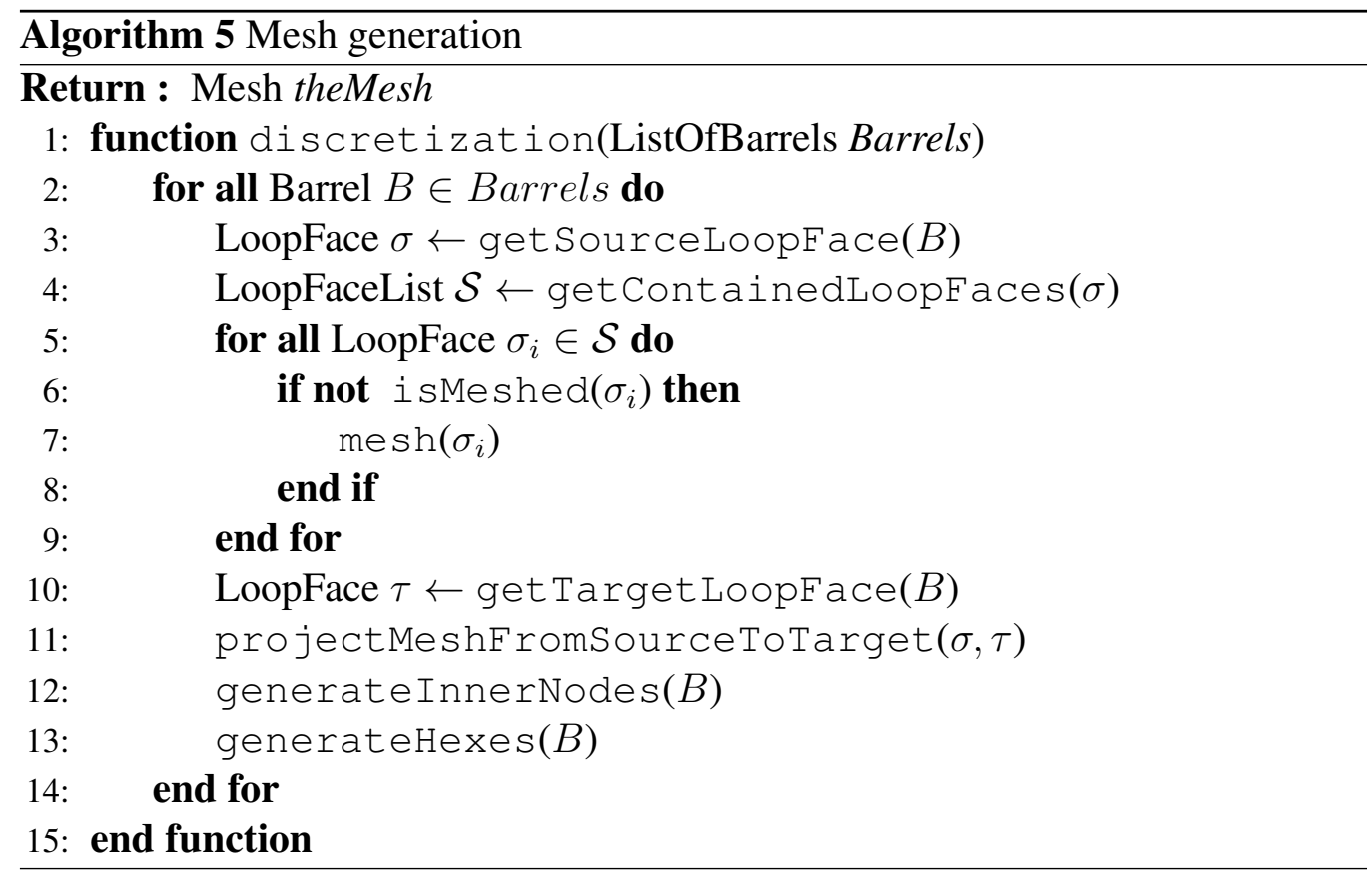

\subsection{One-to-one barrels discretization}

Each barrel can be considered as a one-to-one sweep volume. Thus, we mesh them using the sweep scheme presented in $[5,6,7]$. Algorithm 5 details the discretization process of the barrels. For each barrel, we select the source loop face, $\sigma$, and its corresponding list of loop faces, $\mathcal{S}$, contained in $\sigma$. For each loop face in $\mathcal{S}$ that is not previously meshed, we generate a quadrilateral mesh using the algorithm presented in [20,21]. Then, the mesh generated over $\sigma$ is projected to the target loop face $\tau$. Finally, inner nodes of the barrel are created and hexahedra are generated by connecting consecutive nodes. When all the barrels of each sub-volume are meshed, we have generated a mesh on the whole domain.

\section{Numerical examples}

This section presents five examples of meshes generated using the presented multi-sweeping method. The user prescribes a desired element size and the algorithm automatically decomposes the geometry and discretizes each sub-volume. In the figures that illustrate the examples, the sweep direction is depicted using an arrow. It is worth to notice that to illustrate the behavior of the proposed method we do not smooth the final meshes. 


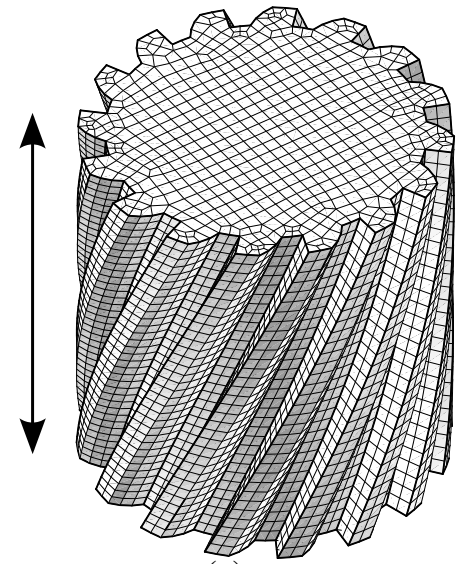

(a)

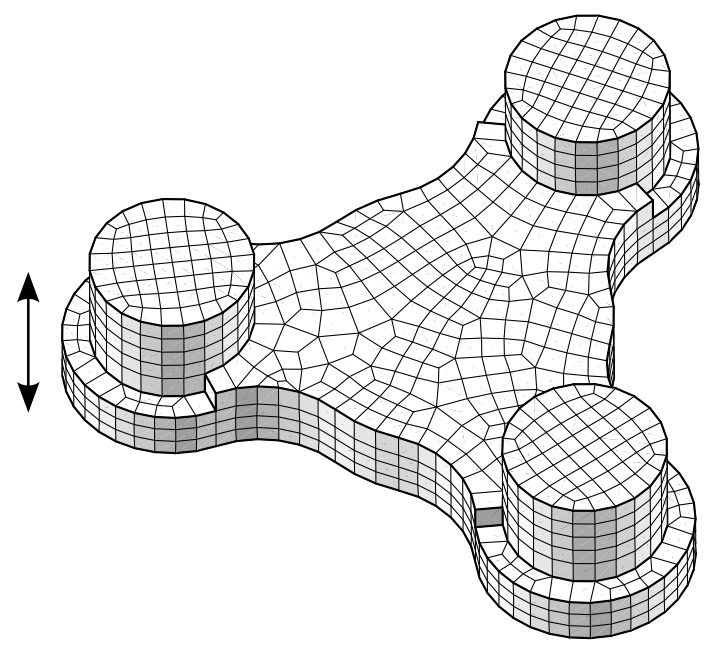

(b)

Figure 18: Meshes generated using the multi-sweeping algorithm. (a) Drill. (b) Mechanical support.

The goal of the first two examples is to show that the proposed algorithm is able to discretize one-to-one and many-to-one sweep volumes as particular cases of many-to-many geometries. To this end, Figure 18(a) presents the mesh generated on a drill and Figure 18(b) presents the mesh generated on a mechanical support.

The third example presents a many-to-many sweep volume which is automatically meshed using the proposed algorithm. The volume is decomposed into two sub-volumes, see Figure 19(a). Then, each sub-volume is meshed separately to generate the final mesh, see Figure 19(b).

In the fourth example we apply the proposed method to discretize a gear. Note that this geometry is a many-to-many extrusion volume delimited by non-planar cap surfaces. Therefore, the inner levels created during the decomposition process are also non-planar and they can lead to a low-quality mesh. Figure 20(a) shows the final mesh generated using the proposed multi-sweeping method. Figure 20(b) shows a detail of the final mesh when the location of inner nodes is obtained by projecting only from target surfaces. Recall that these quadrilateral meshes determine the decomposition of the initial volume into many-to-one sub-volumes. Therefore, they will lead to a low-quality mesh. Instead, Figure 20(c) shows the location of inner nodes when the proposed decomposition process is used. In this 


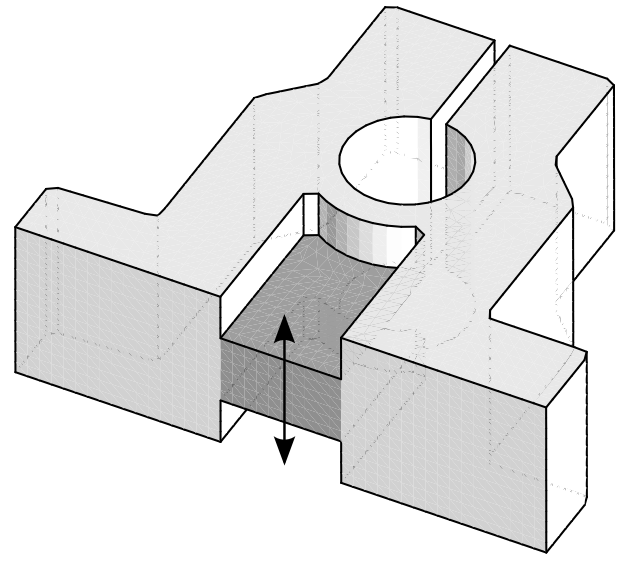

(a)

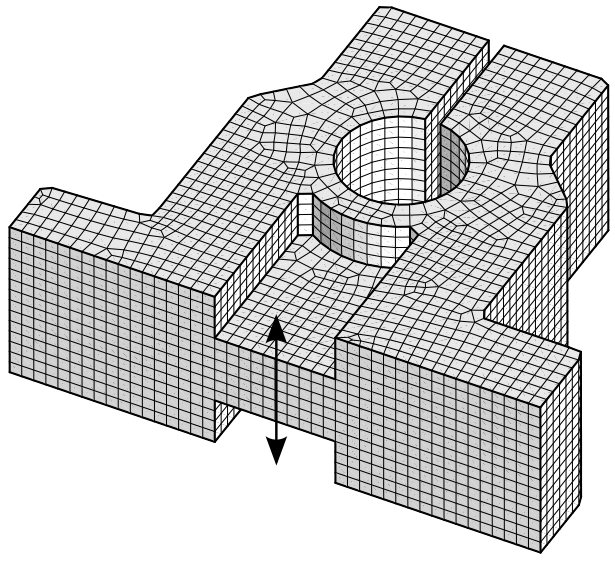

(b)

Figure 19: (a) Mechanical piece decomposition into two sub-volumes. (b) Mesh generated using the multi-sweeping method.

case, the levels of nodes are correctly distributed along the sweep levels. Figure 21(a) shows the distribution of the elements according to the element shape quality [22] when inner nodes are located projecting from target surfaces. Note that there is a large number of elements with quality less than 0.4. Figure 21(b) shows the distribution of the element according to their quality when a weighted interpolation is used. In this case, all the element qualities are greater than 0.5. Table 1 presents a comparison of the statistical information of both meshes. When inner nodes are computed by projecting them form target surfaces, the minimum element quality is zero. This means that there are planar or inverted elements. However, when the proposed method is applied, all the element qualities are above 0.57 . In addition, when our method is applied, the mean of the distribution is increased from 0.76 to 0.86 . Finally, the deviation is decreased when the proposed method is applied.

The fifth example presents the inner nodes created during the decomposition process for a linking rod. In this example, the loops of nodes that define the control loops are non-planar and, in addition, the cap surfaces are curved. Figure 22(a) presents the mesh generated for the linking rod. Figure 22(b) shows the location of inner nodes projecting only from target surfaces. Note that the levels of nodes do not correctly reproduce the shape of the cap surfaces. Figure 22(c) presents the location of inner nodes calculated by the proposed weighted average. In this case, 


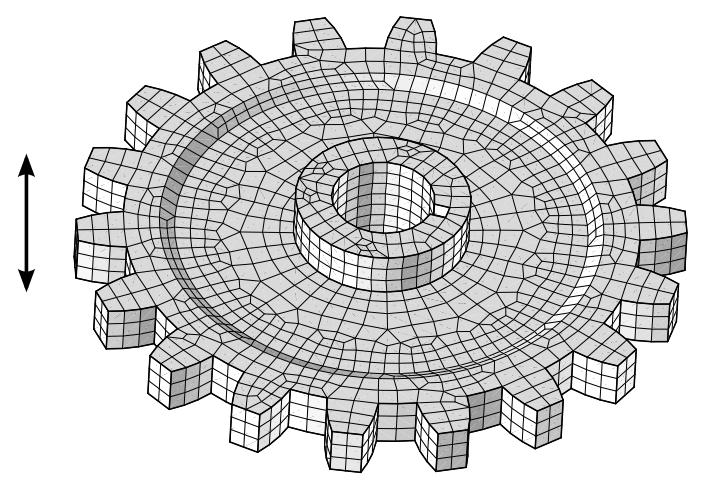

(a)

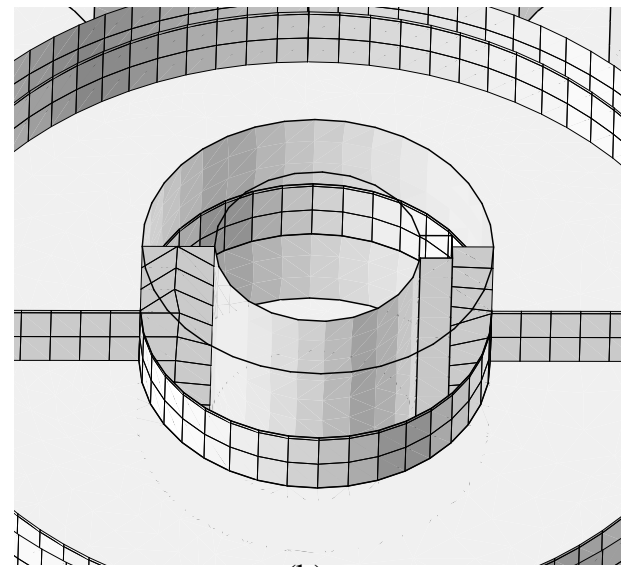

(b)

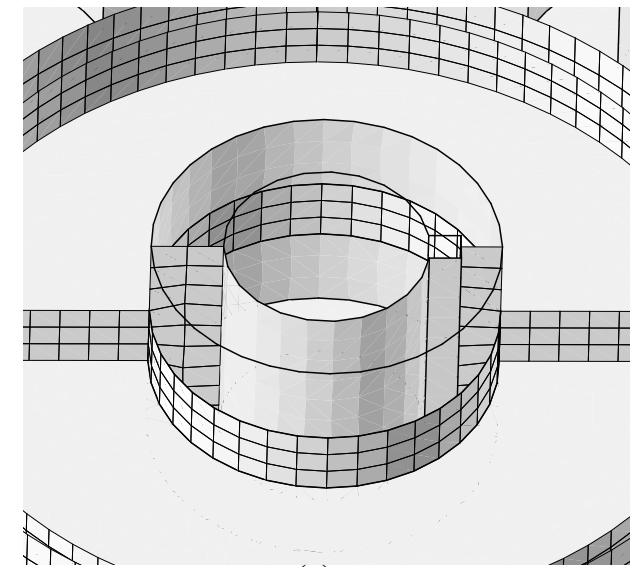

(c)

Figure 20: Location of the inner nodes computed during the decomposition process of the gear. (a) General view of the final mesh. Inner nodes location when: (b) projecting nodes from target surfaces and (c) interpolating nodes projected from source and target surfaces.

Table 1: Element quality statistics of the meshes generated for the gear.

Projecting from Interpolated between

target surfaces source and target surfaces

\begin{tabular}{lll}
\hline $\min \left(f_{\text {shape }}\right)$ & 0.00 & 0.57 \\
$\max \left(f_{\text {shape }}\right)$ & 0.99 & 0.99 \\
$\bar{f}_{\text {shape }}$ & 0.76 & 0.86 \\
$\sigma\left(f_{\text {shape }}\right)$ & 0.23 & 0.09 \\
\hline
\end{tabular}




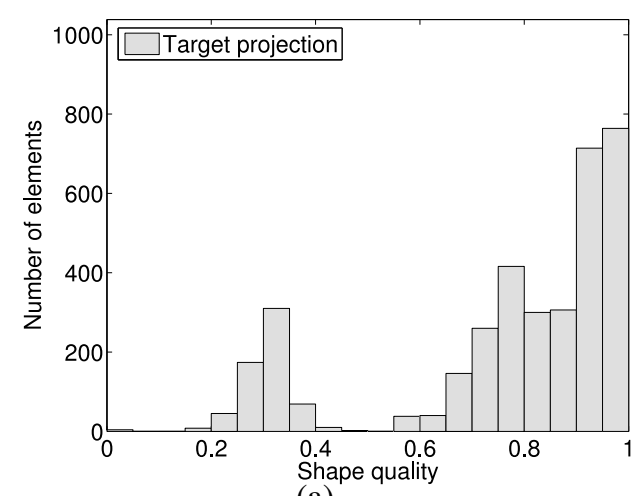

(a)

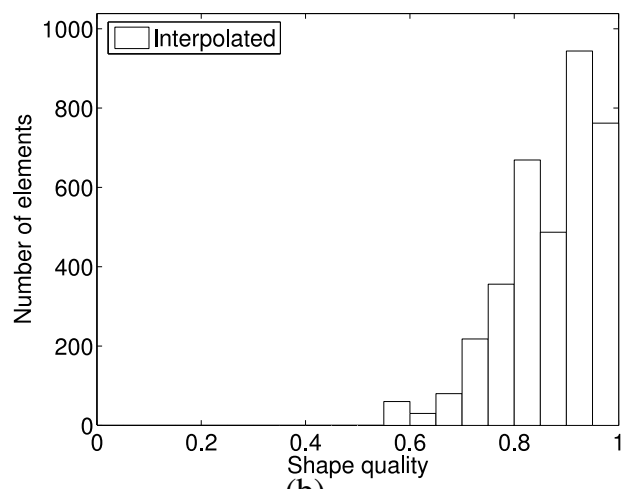

(b)

Figure 21: Distribution of the elements according to their shape quality for the gear meshes. (a) Projecting nodes from target surfaces. (b) Using the proposed weighted average.

Table 2: Element quality statistics of the meshes generated for the linking rod.

\begin{tabular}{lcc}
\hline & $\begin{array}{c}\text { Projecting from } \\
\text { target surfaces }\end{array}$ & $\begin{array}{c}\text { Interpolated between } \\
\text { source and target surfaces }\end{array}$ \\
\hline $\min \left(f_{\text {shape }}\right)$ & 0.00 & 0.37 \\
$\max \left(f_{\text {shape }}\right)$ & 0.99 & 0.99 \\
$\bar{f}_{\text {shape }}$ & 0.82 & 0.85 \\
$\sigma\left(f_{\text {shape }}\right)$ & 0.13 & 0.10 \\
\hline
\end{tabular}

the node levels smoothly follow the shape of cap surfaces. Figure 23(a) shows the distribution of the element according to their quality when the inner nodes are located projecting from target surfaces. Note that there are elements with quality equal to zero. Figure 23(b) presents the distribution of the element according to their quality when inner nodes are located using the proposed weighted average. In this case, a better quality distribution is obtained. Table 2 presents a comparison of the statistical information of both meshes. When the inner nodes are computed projecting from target faces, the minimum element quality is 0.0. Instead, our method provides a minimum element quality of 0.37 . The maximum element quality is similar in both meshes. Using the proposed method, the mean of the distribution is slightly increased and the deviation is slightly decreased. 


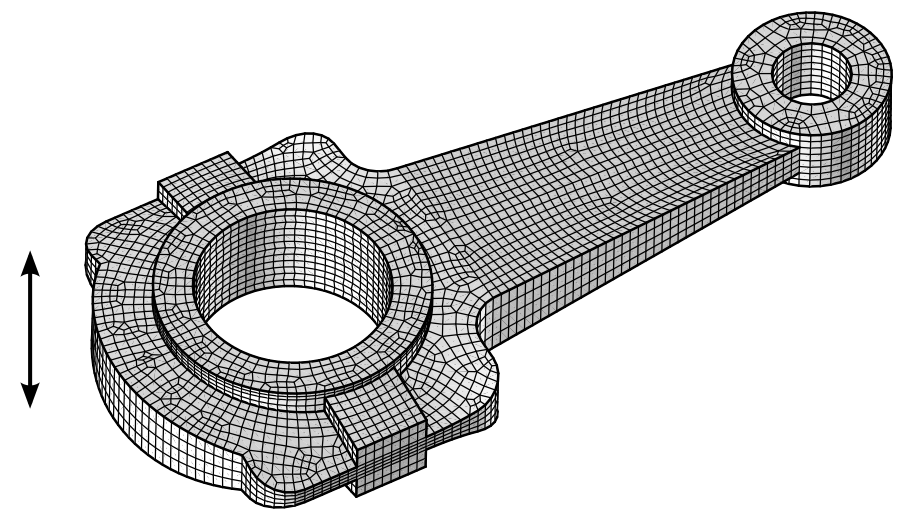

(a)

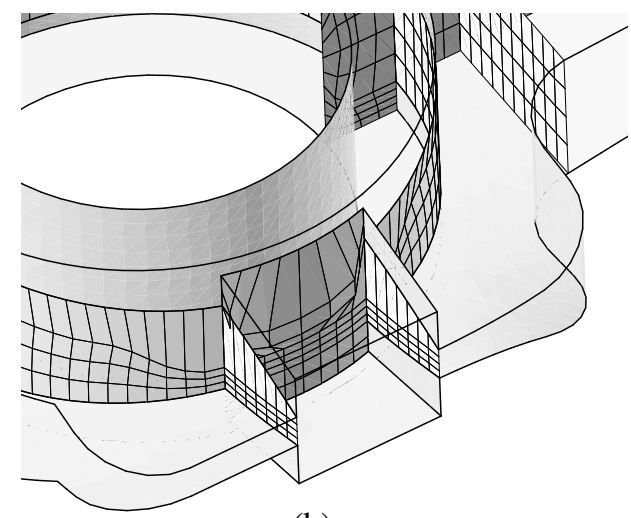

(b)

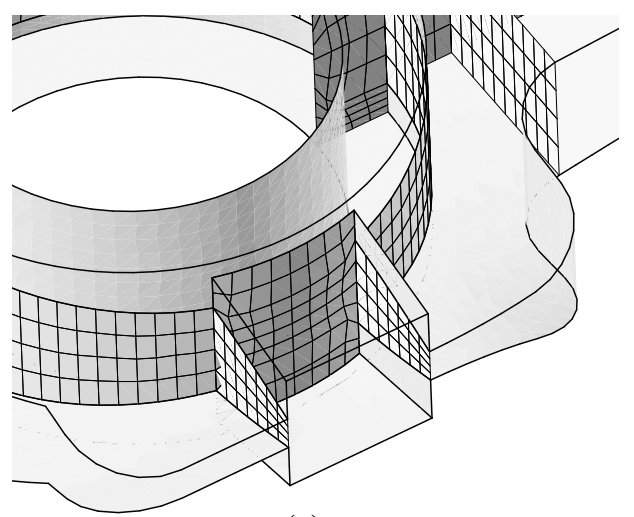

(c)

Figure 22: Location of the inner nodes computed during the decomposition process of the linking rod. (a) General view of the final mesh. Inner nodes location when: (b) projecting nodes from target surfaces and (c) interpolating nodes projected from source and target surfaces.

\section{Conclusions}

We have presented a multi-sweeping method to decompose and mesh manyto-many sweep volumes. We have pointed that the quality of the final mesh depends on the the robustness of the imprinting process. Therefore, we have developed a new algorithm to increase the robustness of the computed imprints. This algorithm is based on a planar representation of each control loop called computational domain. We use this planar representation for two purposes. On the one hand, we use it to perform all the imprints between surfaces. Since the computational domain is planar, the robustness of the imprinting process is increased. On the other hand, the computational domain is also used to project nodes from a 


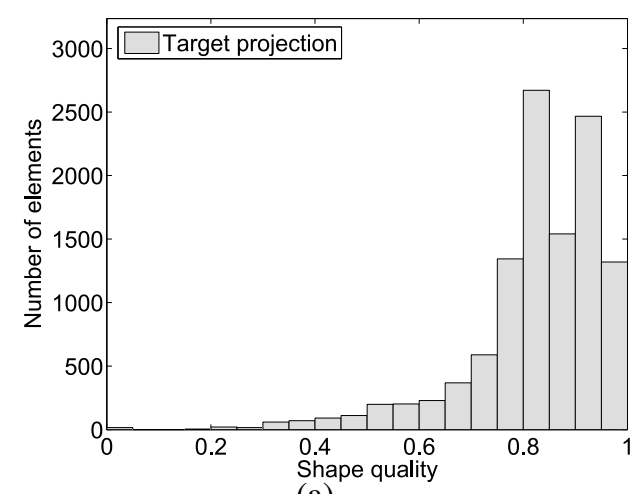

(a)

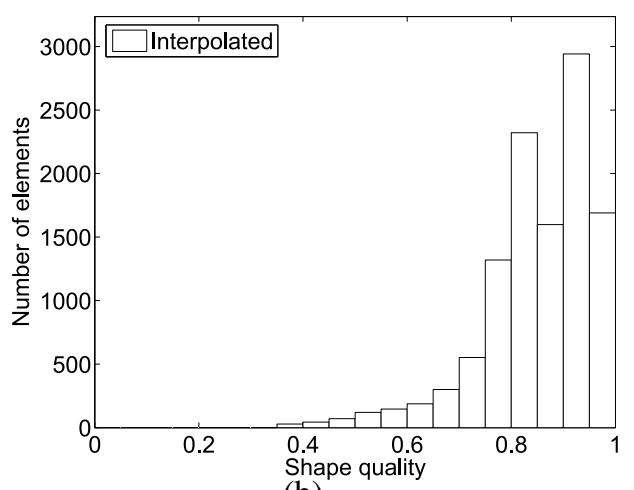

(b)

Figure 23: Distribution of the elements according to their shape quality for the linking rod meshes. (a) Projecting nodes from target surfaces. (b) Using the proposed weighted average.

propagated target loop face onto geometric source surfaces. In fact, we determine this projection by means of a least-squares approximation of an affine mapping. This mapping is defined between the $2 \mathrm{D}$ representation of the source surface in the computational domain and its representation in the parametric space.

In addition, the proposed method performs a three-stage procedure to improve the position of inner nodes created during the decomposition process. In the first stage, the sweep nodes on target surfaces are projected towards source surfaces and the decomposition of the volume is computed. However, the location of these inner nodes may lead to meshes with low-quality elements. In the second stage, the nodes on source surfaces are projected back towards target surfaces. In the third stage, the final location of inner nodes is computed as a weighted average of the position of nodes projecting from the target and the source surfaces. Note that this technique is based on the inner node creation process for the classical sweeping methods. That is, the final position of inner nodes created during the decomposition process is the average of the projection from source and target faces. Thus, inner nodes follow the shape of both target and source surfaces.

An additional novelty of the proposed method is that we do not have imposed an even number of intervals during the imprinting process. Therefore, the quality of the imprints is improved. Instead, this condition is only imposed during the discretization process by solving an integer linear problem. Actually, we impose an even number of intervals only on the boundary of source loop faces obtained during the imprinting process. Thus, the size of the integer linear problem is reduced. The number of intervals of the boundary of source loop faces is then 
propagated along the sweep direction.

Several examples have been presented to show the capabilities and robustness of the proposed multi-sweeping method. Finally, it is worth to notice that the multi-sweeping method is successfully implemented in the ez4u meshing environment [23].

\section{Acknowledgements}

This work was partially sponsored by the Spanish Ministerio de Ciencia e Innovación under grants DPI2007-62395, BIA2007-66965 and CGL2008-06003C03-02/CLI.

\section{References}

[1] J. F. Thompson, Handbook of Grid Generation, CRC Press, 1999.

[2] P. Frey, P. George, Mesh Generation: Application to Finite Elements, Wiley, 2008.

[3] P. Knupp, Next-generation sweep tool: a method for generating all-hex meshes on two-and-one-half dimensional geometries, in: Proceedings of the 7th International meshing roundtable, 1998.

[4] M. L. Staten, S. A. Canann, S. J. Owen, BMSweep: Locating interior nodes during sweeping, Engineering with Computers 15 (1999) 212-218.

[5] X. Roca, J. Sarrate, A. Huerta, Mesh projection between parametric surfaces, Communications in Numerical Methods in Engineering 22 (2006) 591-603.

[6] X. Roca, J. Sarrate, A new least-squares approximation of affine mappings for sweep algorithms, Engineering with Computers 26 (2010) 327-337.

[7] X. Roca, J. Sarrate, An automatic and general least-squares projection procedure for sweep meshing, Engineering with Computers 26 (4) (2010) 391406.

[8] M. A. Scott, S. E. Benzley, S. J. Owen, Improved many-to-one sweeping, International Journal for Numerical Methods in Engineering 63 (2006) 332348. 
[9] D. R. White, S. Saigal, S. Owen, CCSweep: an automatic decomposition of multi-sweep volumes, Engineering with Computers 20 (2004) 222-236.

[10] T. Blacker, The Cooper tool, in: Proceedings of the 5th International meshing roundtable, 1996.

[11] M. Lai, S. Benzley, D. R. White, Automated hexahedral mesh generation by generalized multiple source to multiple target sweeping, International Journal for Numerical Methods in Engineering 49 (2000) 261-275.

[12] J. Shepherd, S. A. Mitchell, P. Knupp, D. R. White, Methods for Multisweep Automation, in: Proceedings of the 9th International Meshing Roundtable, 2000 .

[13] D. R. White, T. Tautges, Automatic scheme selection for toolkit hex meshing, International Journal for Numerical Methods in Engineering 49 (2000) $127-144$.

[14] D. R. White, Automatic quadrilateral and hexahedral meshing of pseudocartesian geometries using virtual subdivision, 1996.

[15] E. Ruiz-Gironés, J. Sarrate, Generation of structured meshes in multiply connected surfaces using submapping, To appear in Advances in Engineering Software .

[16] D. White, S. Saigal, Improved imprint and merge for conformal meshing, in: Proceedings of the 11th International Meshing Roundtable, 2002.

[17] J. O'Rourke, Computational geometry in C, Cambridge University Press, 1998.

[18] A. Schrijver, Theory of Linear and Integer Programming, John Wiley and Sons, 1998.

[19] M. Berkelaar, LP Solve, http://sourceforge.net/projects/lpsolve, 2009.

[20] J. Sarrate, A. Huerta, Efficient unstructured quadrilateral mesh generation, International Journal for Numerical Methods in Engineering 49 (2000) $1327-1350$. 
[21] J. Sarrate, A. Huerta, Automatic mesh generation of nonstructured quadrilateral meshes over curved surfaces in $\mathbb{R}^{3}$, in: Proceedings of the 3rd European Congress on Computational Methods in Applied Sciences and Engineering, ECCOMAS, 2000.

[22] P. M. Knupp, Algebraic mesh quality metrics for unstructured initial meshes, Finite Elements in Analysis and Design 39 (2004) 217-241.

[23] X. Roca, J. Sarrate, E. Ruiz-Gironés, A graphical modeling and mesh generation environment for simulations based on boundary representation data, in: Congresso de Métodos Numéricos em Engenharia, 2007. 